\title{
A Peeling Approach for Integrated Manufacturing of Large Mono-Layer h-BN Crystals
}

Ruizhi Wang ${ }^{\dagger}$, David G. Purdie ${ }^{\dagger \dagger}$, Ye Fan ${ }^{\dagger}$, Fabien C-P. Massabuau ${ }^{\S}$, Philipp BraeuningerWeimer $^{\dagger}$, Oliver J. Burton ${ }^{\dagger}$, Raoul Blumell, Robert Schloegl ${ }^{\perp}$, Antonio Lombardo ${ }^{\dagger, \ddagger}$, Robert S. Weatherup ${ }^{\nabla, 0}$, Stephan Hofmann* ${ }^{\dagger}$

${ }^{\dagger}$ Department of Engineering, University of Cambridge, 9 JJ Thomson Avenue, Cambridge CB3 0FA, United Kingdom

Cambridge Graphene Centre, University of Cambridge, 9 JJ Thomson Avenue, Cambridge CB3 0FA, United Kingdom

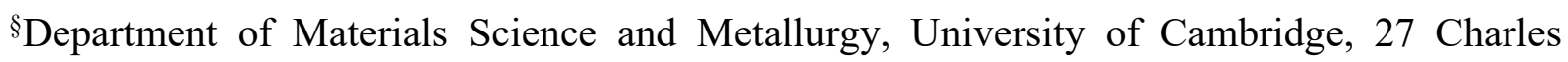
Babbage Road, Cambridge CB3 0FA, United Kingdom

"Helmholtz-Zentrum Berlin für Materialen und Energie, D-12489 Berlin, Germany

${ }^{\perp}$ Fritz Haber Institute, D-14195 Berlin-Dahlem, Germany

${ }^{\nabla}$ School of Chemistry, University of Manchester, Oxford Road, Manchester M13 9PL, UK

OUniversity of Manchester Harwell Campus, Diamond Light Source, Didcot, Oxfordshire, OX11 0DE, UK

\section{Corresponding Author}

*Stephan Hofmann: Emailsh315@cam.ac.uk 


\begin{abstract}
Hexagonal boron nitride (h-BN) is the only known material aside from graphite with a structure composed of simple, stable, non-corrugated atomically thin layers. While historically used as lubricant in powder form, h-BN layers have become particularly attractive as an ultimately thin insulator, barrier or encapsulant. Practically all emerging electronic and photonic device concepts rely on h-BN exfoliated from small bulk crystallites, which limits device dimensions and process scalability. We here focus on a systematic understanding of Pt catalysed h-BN crystal formation, in order to address this integration challenge for mono-layer h-BN via an integrated chemical vapour deposition (CVD) process that enables h-BN crystal domain sizes exceeding $0.5 \mathrm{~mm}$ and a merged, continuous layer in a growth time less than $45 \mathrm{~min}$. The process makes use commercial, reusable Pt foils, and allows a delamination process for easy and clean h-BN layer transfer. We demonstrate sequential pick-up for the assembly of graphene/h-BN heterostructures with atomic layer precision, while minimizing interfacial contamination. The approach can be readily combined with other layered materials and enables the integration of CVD h-BN into high quality, reliable 2D material device layer stacks.
\end{abstract}

Keywords: h-BN, 2D materials, CVD, transfer, catalyst, heterostructures, graphene, platinum 
Scalable manufacture remains a central challenge in the application of two-dimensional layered materials (2DLMs). In recent years, major advances have been made regarding chemical vapour deposition (CVD) of 2DLMs such as graphene $(\mathrm{Gr})^{1,2}$ and hexagonal boron nitride (hBN). ${ }^{3,4}$ Many studies have revealed the details of the growth processes on select, generally catalytic, substrates. $^{5-7}$ The focus thereof has been on achieving ever-larger single-crystalline regions by lowering the nucleation density ${ }^{8,9}$ and/or by merging aligned domains. ${ }^{10,11}$ However, emerging applications require integration into device stacks. The most versatile route for this is 2DLM transfer from the CVD growth catalyst to the designated device, often including the vertical stacking of 2DLMs to form van der Waals heterostructures. A number of transfer methods have been proposed for CVD Gr and h-BN, including wet transfer, ${ }^{12,13}$ dry transfer, ${ }^{14-}$ 16 electrochemical delamination ${ }^{17-19}$ and lift-off-transfer. ${ }^{20}$ Progress in developing these methods has not kept pace with large-area 2DLM crystal growth, and the introduction of contamination and damage remain major constraints, which is exceptionally severe in case of heterostructures that rely on atomically clean interfaces..$^{21,22}$

Heterostructure devices fabricated entirely using exfoliated 2DLM now achieve carrier mobility values close to theoretically-predicted limits. ${ }^{23-25}$ As exfoliation is an inherently nonscalable approach to fabrication, significant efforts have been made to replace each of the constituent layers using CVD 2DLMs in a step-wise manner. ${ }^{26,27}$ The approach for high mobility Gr channels has been to use h-BN flakes exfoliated from bulk crystallites to peel off CVD Gr from the growth substrate, thus minimising transfer related defects and interface contamination. Using dry transfer, $\mathrm{Cu}$ catalysed CVD Gr has been shown to exhibit electron and hole mobilities well above $50,000 \mathrm{~cm}^{2} / \mathrm{Vs}$ at room temperature ${ }^{26}$ and ballistic transport lengths of $28 \mu \mathrm{m}$ have been demonstrated at temperatures below $2 \mathrm{~K}$, limited solely by device dimensions, i.e. the size of exfoliated h-BN flakes. ${ }^{27}$ While highlighting the potential for 
applications particularly in optoelectronics and sensing, these examples still rely on mechanical exfoliation from bulk h-BN crystallites, giving limited, random flake size and varying thickness.

Our motivation here is to address the current challenge of integrating CVD h-BN into such 2DLM heterostructures, particularly using the transfer route. This demands holistic process development, as not only large h-BN mono-layer crystals are required, but also their viable, clean transfer away from the growth catalyst. We focus on Pt as catalyst, which has a lower vapour pressure and higher melting point ${ }^{28}$ than widely employed $\mathrm{Cu},{ }^{7,12}$ hence allows a wider high temperature parameter space and at the same time minimises potential metal contamination particularly of the growth reactor. Reported CVD h-BN domain sizes on Pt to date are typically only a few $\mu \mathrm{m},{ }^{29,30}$ and 2DLM transfer away from the Pt surface currently relies on electrochemical delamination. While previous studies have focussed on the h-BN/Pt surface interactions, ${ }^{31,32}$ we here establish a first order understanding of the underlying growth mechanisms which hitherto remained unclear. ${ }^{37}$ Starting from commercial poly-crystalline $\mathrm{Pt}$ foils, this understanding allows us to devise an integrated CVD process that includes rapid, borazine induced Pt foil crystallisation and separate control of h-BN nucleation density and domain/film growth, to enable monolayer h-BN domains with lateral sizes exceeding $0.5 \mathrm{~mm}$ at relatively short growth times. Importantly, as-grown h-BN mono-layers can be easily and cleanly transferred using an entirely delamination-based approach, which also enables the reuse of the substrate. The latter is pertinent considering the high cost of Pt. We demonstrate sequential pick-up to create h-BN films of controlled layer thicknesses and graphene/h-BN heterostructures, while minimizing interfacial contamination and showing high device performance. These results enable the integration of CVD h-BN in high quality 2DLM heterostructures. 


\section{Results}

Fig. 1 gives an overview of our CVD process and how h-BN growth proceeds. In its basic form, our process, which we refer to as a sequential step growth (SSG), consists of two coupled borazine exposures at different pressures (Fig. 1a). In contrast, widely used single exposure at fixed temperature and pressure is referred to as "standard" growth (SG). We use borazine $\left(\mathrm{B}_{3} \mathrm{H}_{6} \mathrm{~N}_{3}\right)$ as a combined boron (B) and nitrogen $(\mathrm{N})$ precursor, which is isostructural to benzene and has a high vapour pressure of 340 mbar at room temperature. ${ }^{33}$ In conjunction with our cold-wall CVD reactor system (base pressure $<2 \times 10^{-6} \mathrm{mbar}$, further details in Experimental Methods), a well-controlled precursor atmosphere can be maintained, in contrast to the use of ammonia borane, which can exhibit a complex and evolving decomposition profile especially for hot-wall CVD reactor conditions. ${ }^{34}$ Our main findings relate to fundamental interactions with the catalytic growth substrate and hence are transferable to other $\mathrm{B}$ and $\mathrm{N}$ precursors. In particular, we note that while borazine represents a precursor with pre-defined stoichiometry $(\mathrm{B}: \mathrm{N}=1)$, it dissociates on the Pt surface. This means that the interaction of the constituent elements with the catalyst will dictate the actual supply of the elements during CVD. ${ }^{7}$ We use commercially available polycrystalline Pt foils (25 $\mu \mathrm{m}, 99.99 \%$, Alfa Aesar $)$ as growth substrates, which are remotely heated using an IR laser with a beam shaper that creates a homogeneous field with a top-hat profile. The laser heating enables a low thermal mass for fast ramping to and from growth temperatures $\left(\mathrm{T}_{\mathrm{gr}}\right)$ of up to $1300{ }^{\circ} \mathrm{C}$, and at the same time minimises reactor cross-contamination effects. For the given set-up we see no laser specific effects here other than heating. If not stated otherwise, all SEM images are taken immediately after growth with around $30 \mathrm{~min}$ or less atmospheric exposure for transfer. This is important 
as the secondary electron (SE) contrast for h-BN mono-layers can change on $\mathrm{Pt}$, as we will discuss below. Further details are given in the Experimental Section.

The first exposure in SSG (Fig. 1a), at relatively high borazine pressure $\left(\mathrm{P}_{\mathrm{sd}}\right)$, promotes recrystallization and grain growth of the Pt foil which is initially highly poly-crystalline (Fig. 1b). The h-BN nucleation density and homogeneity are then controlled by briefly removing the precursor during the homogenization stage (thomo; see Fig. 1a), which leads to the dissolution and removal of excess $\mathrm{h}-\mathrm{BN}$. In the second exposure phase $\left(\mathrm{t}_{\exp }\right)$ the nuclei are then laterally expanded into large mono-layer h-BN crystals via exposure at low borazine pressure $\left(\mathrm{P}_{\exp }\right)$. Upon further exposure, these merge into a continuous h-BN mono-layer film. The h-BN monolayers thus grown can then be delaminated directly from the Pt, which can be reused for further growth (see SI, Fig. S9).

It remains unclear from previous literature on Pt catalysed h-BN CVD whether growth occurs isothermally or via precipitation on cooling. ${ }^{30}$ Hence, we make use of in situ and ex situ characterisation to establish a first order understanding of the underlying growth mechanisms as basis for further process development. In situ X-ray photoelectron spectroscopy (XPS) provides surface-sensitive information on the growth mechanism of h-BN (see experimental section for details). ${ }^{4,7}$ Fig. 2 shows in situ XPS measurements of a Pt foil during a basic onestep borazine exposure (process diagram Fig. 2a). XPS measurements are taken throughout the CVD process, i.e. during heating to $\mathrm{T}_{\mathrm{gr}}$, subsequent borazine exposure at constant pressure $\mathrm{p}_{\mathrm{sd}}$ and cool-down. Fig. $2 \mathrm{~b}$ shows the evolution of B1s and N1s core-level spectra taken during borazine exposure $\left(3 \times 10^{-4}\right.$ mbar), at a $\mathrm{T}_{\mathrm{gr}}$ of $1100^{\circ} \mathrm{C}$ (step II.1 - II.2). Both the B1s and N1s spectra show the emergence of a peak that grows in intensity with time, consistent with the isothermal growth of h-BN on the Pt surface. XPS spectra measured toward the end of borazine 
exposure show a main B1s peak centred at a binding energy of $\sim 191.5 \mathrm{eV}$ and N1s peak centred at $\sim 399.0 \mathrm{eV}$ (Fig. 2c). We observe the $\pi \rightarrow \pi *$ plasmon shake up satellite at $\sim 200 \mathrm{eV}$ corresponding to $\mathrm{sp}^{2}$ bonded h-BN, which can be more clearly discerned when multiple spectra are summed to improve the signal to noise ratio (see SI, Fig. S2). Analysis of the relative peak intensities also confirms that the B:N ratio is $\sim 1$. Based on ex situ measurements using transmission electron microscopy (TEM) and Raman spectroscopy (see below and SI), we exclude significant formation of cubic BN and multilayer h-BN. Hence we assign the given XPS signatures to mono-layer h-BN, consistent with previous literature. ${ }^{7,35,36}$

After the first exposure step in SSG and when removing the precursor (temperature kept at $\mathrm{T}_{\mathrm{gr}}$ $\left.=1100{ }^{\circ} \mathrm{C}\right)$, the B1s and N1s peaks disappear within less than 2 minutes (Fig. 2c). The B1s and N1s peaks do not reappear upon cooling at these conditions. There are multiple possible explanations for the disappearance of the Pt supported h-BN mono-layer. One process that has been reported in the context of removing Gr grown on $\mathrm{Pt}$ is etching with $\mathrm{H}_{2}$ at $1060{ }^{\circ} \mathrm{C} .{ }^{37}$ Other studies have investigated the stability of $\mathrm{h}-\mathrm{BN}$ on $\mathrm{SiO}_{2}$, a non-catalytic substrate, in an oxygen containing atmosphere and found the onset of degradation at $850{ }^{\circ} \mathrm{C}^{38}$ and complete removal at $1000{ }^{\circ} \mathrm{C} .{ }^{39}$ The stability of h-BN may be further reduced when on a catalyst such as the growth substrate, as in the case of $\mathrm{h}-\mathrm{BN}$ on $\mathrm{Cu}$ where depending on the state of oxygen intercalation, the h-BN can dissociate completely at $700{ }^{\circ} \mathrm{C} .{ }^{7}$ Given the absence of any gas after precursor removal and a base pressure of below $1 \times 10^{-8}$ mbar of the in situ XPS chamber, we exclude the possibility of significant etching for our experiments. It should be noted however, that typical CVD reactors, including our custom-built cold-wall CVD system (base pressure $<2 \times 10^{-6} \mathrm{mbar}$ ), have a higher base pressures, and thus we cannot fully exclude the contribution of etching through residual gases. Nonetheless, we suggest that the main mechanism of removal here is that at the given growth temperatures $\mathrm{h}-\mathrm{BN}$ is not stable on the growth catalyst 
once the precursor has been removed, and either desorbs, is dissolved into the bulk or both. The bulk solubility of $\mathrm{B}$ in $\mathrm{Pt}$ is reported to be $\sim 2.5$ at $\%$ at $\mathrm{T}_{\mathrm{gr}}=1100{ }^{\circ} \mathrm{C} .{ }^{40} \mathrm{We}$ could not find any relevant ternary phase diagrams in literature, or reliable data on $\mathrm{N}$ solubility in $\mathrm{Pt}$, but it has previously been assumed that the $\mathrm{N}$ solubility in bulk $\mathrm{Pt}$ is negligible. ${ }^{29}$ Simple thermodynamic bulk solubility considerations are typically inadequate for describing growth of nanomaterials where kinetic processes and local supersaturations are often dominant. ${ }^{41}$ Furthermore, under certain conditions (see SI, Fig. S4) we have observed precipitation effects indicating not only boron (see also Fig. 3) but also nitrogen solubility, consistent with the hBN layers dissolving into the catalyst bulk (Fig. 2c).

As well as revealing the presence and evolution of $\mathrm{h}-\mathrm{BN}$ on the Pt surface, we are also able to monitor the chemical state of the Pt catalyst with in situ XPS. Fig. $2 \mathrm{~d}$ presents the Pt $4 \mathrm{f}$ core level spectra for bare (prior to borazine exposure) and h-BN covered Pt surfaces at two different X-ray excitation energies taken at $\mathrm{T}_{\mathrm{gr}}=950{ }^{\circ} \mathrm{C}$. Photoelectrons collected with higher X-ray energy have longer inelastic mean free paths $\left(\lambda_{\text {IMFP }} \approx 4.5 \AA\right.$ at $225 \mathrm{eV}, \lambda_{\text {IMFP }} \approx 7.6 \AA$ at $525 \mathrm{eV}),{ }^{42}$ and thus higher information depth, i.e. the data represents depth resolved information about the catalyst surface composition. We observed no changes in the Pt4f spectrum compared to the clean surface indicating the absence of any significant $\mathrm{B}$ and $\mathrm{N}$ phases near the surface.

The results for CVD of h-BN on Pt are consistent with a first-order kinetic growth model previously introduced for other transition metal catalysts, which takes into account precursor and elemental flux balances. ${ }^{7,41}$ The key findings regarding the optimized CVD process in Fig. 1 are that h-BN grows isothermally on Pt and no further growth occurs upon cooling due to precipitation. This is in contrast h-BN growth on Fe, where the contribution to growth due to precipitation is significant and thus control thereof is crucial for growth of large grain size h- 
BN. ${ }^{4}$ At the chosen CVD conditions, h-BN growth appears to be driven by a local B and N supersaturation at the surface. At high temperatures $\left(\mathrm{T}_{\mathrm{gr}}=1100^{\circ} \mathrm{C}\right.$, Fig. $\left.2 \mathrm{c}\right)$ the Pt supported h-BN mono-layer film dissociates and at least partly dissolves into the Pt bulk once the borazine flux is removed. Within the parameter space covered by our in and ex situ experiments (max. borazine pressure of $10^{-2}$ mbar; max. temperature of $1400^{\circ} \mathrm{C}$; max. exposure time of $30 \mathrm{~min}$ ) we only observe monolayer h-BN on Pt. This is independent of the cooling rate which we have varied from $10{ }^{\circ} \mathrm{C} / \mathrm{min}$ to immediate quenching (temperature drop of $>500^{\circ} \mathrm{C}$ in a period of $\sim 5 \mathrm{~s})$. This is in contrast to h-BN growth on Fe, where multi-layer h-BN formation is observed. ${ }^{4}$

Fig. 3 shows the effect of annealing of the Pt foil and how the choice of CVD atmosphere prior to growth impacts its texture and crystallinity. h-BN growth is linked to the nature of the growth substrate, analogous to graphene CVD. Different catalyst foil facets show different catalytic growth activities and lead to varying 2DLM nucleation density, domain alignment and domain shape evolution. ${ }^{43}$ Hence optimising the crystallinity of the catalyst foil is an important part of the CVD process. A first indication of the crystallinity can be obtained through SEM. Crystal domains with different orientations will differ in brightness due to channelling contrast. The lack thereof gives a strong indication of a single Pt crystal facet. We carried out a systematic set of experiments with commercial Pt foils that were annealed in vacuum for $15 \mathrm{~min}$, similar to the general SSG process outlined in Fig. 1, and then subjected to an additional 2 min of annealing under different gas environments (Fig. 3a). For annealing in vacuum (at base pressure $\left.<2 \times 10^{-6} \mathrm{mbar}\right), \mathrm{H}_{2}\left(10^{-3} \mathrm{mbar}\right)$ or $\mathrm{NH}_{3}\left(10^{-3} \mathrm{mbar}\right)$, SEM shows a binary distribution of grain sizes. The majority of the Pt grains have a lateral size around $50 \mu \mathrm{m}-100 \mu \mathrm{m}$ (Fig. 3b, Loc. 1). A few selected Pt grains grow significantly larger, reaching millimetre-scale (Fig. 3b, Loc. 2). In contrast, when annealed in borazine for $2 \mathrm{~min}$, at otherwise identical conditions, the Pt surface shows uniform SEM contrast across a few $\mathrm{mm}^{2}$ of sample area, indicative of a 
dominant single in-plane orientation (Fig. 3b). No evidence of h-BN growth is observed at this stage. X-ray diffraction measurements (XRD) were taken to investigate the Pt foil texture in

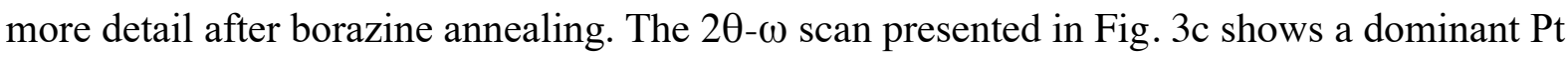
(1 111 ) orientation following annealing. This is to be expected given that $\left(\begin{array}{lll}1 & 1 & 1\end{array}\right)$ is the lowest energy interface for FCC crystals, such as Pt. The texture map in Fig. 3d of the Pt $\left(\begin{array}{lll}1 & 1 & 1\end{array}\right)$ reflection (at $2 \theta=39.73^{\circ}$ ) shows one pole in the symmetric position $\left(\chi \sim 0^{\circ}\right)$ and 3 poles at $\chi \sim$ $70^{\circ}$ and spaced by $\phi=120^{\circ}$ from each other. This is evidence that the vast majority of the $\mathrm{Pt}$ grains have the same orientation, i.e. the grains are not rotated relative to each other. The foil is not completely single-crystalline as highlighted by the additional weaker poles, for example the one at $\chi \sim 70^{\circ}$ and $\phi=90^{\circ}$, which indicate that there are some grains, albeit a minority (potentially in the bulk of the foil), that are rotated with respect to the dominant orientation. These results are in agreement with additional measurement taken using electron backscatter diffraction (EBSD), given in Fig. S15 in the supplementary information. We note that the Pt surface roughness is not significantly altered by this crystallisation, with an average roughness measured by atomic force microscopy (AFM) of $\mathrm{R}_{\mathrm{a}}=5.3 \mathrm{~nm}$ for the poly-crystalline and $\mathrm{R}_{\mathrm{a}}=$ $4.7 \mathrm{~nm}$ for the crystallized foil (see SI, Fig. S11). We thus exclude a change of surface roughness as the cause for variations in the h-BN growth.

There are a number of reports on thermal recrystallization of $\mathrm{Cu}$ foils for improved graphene CVD, using extended thermal pre-treatment ${ }^{44,45}$ and thermal gradients. ${ }^{11} \mathrm{We}$ also explore thermal gradients (see SI, Fig. S1), and emphasize that longer annealing times lead to improved foil crystallisation. Here, however, we focus on the accelerated crystallisation observed for borazine exposure which has benefits as part of an integrated process. Upon thermal annealing, recrystallization is at first driven by a reduction of dislocation energy, followed by grain growth driven by the minimisation of the overall energy associated with grain-boundaries (GB). The 
latter involves normal grain growth where all existing grains grow uniformly, as well as abnormal grain growth (also known as secondary crystallization) where one type of grain will grow significantly faster than the others driven by the difference in surface energies between different grain orientations. ${ }^{46-50}$ Our data shows that such abnormal growth in Pt is significantly enhanced by the presence of borazine. For Pt thin films it has been frequently observed that the presence of oxygen is detrimental to the formation of grains with $\left(\begin{array}{lll}1 & 1 & 1\end{array}\right)$ surface orientation. ${ }^{47,51}$ In this context $\mathrm{B}$, which can readily adsorb in Pt grain boundaries, ${ }^{52}$ is frequently used as deoxidizer, ${ }^{53}$ and the addition of $\mathrm{B}$ to Pt has been seen to cause GB unpinning. ${ }^{50}$ We therefore attribute the accelerated abnormal Pt grain growth to GB unpinning via removal of pre-existing solutes. Another potential mechanism could be B causing solute drag by decorating and pinning selective GBs, leaving only high mobility GBs able to grow.

Having established the rationale for the catalyst foil crystallisation step, we now consider each subsequent step in the SSG process (Fig. 1a), i.e. seeding, homogenization and domain expansion. We carried out a systematic set of SG experiments and focus on the role of key parameters, particularly exposure times $\left(t_{s d}, t_{\text {homo }}, t_{\exp }\right)$, exposure pressures $\left(\mathrm{P}_{\text {sd }}, \mathrm{P}_{\text {exp }}\right)$ and temperatures $\left(\mathrm{T}_{\mathrm{gr}}\right)$, for each individual step of the integrated SSG process. In Fig. 4, a series of SEM images show the outcome of SG growth experiments at precursor pressures $\left(\mathrm{P}_{\mathrm{sd}}=1 \times 10^{-5}\right.$ mbar) corresponding to those used in the first SSG step (Fig. 1a). The topmost images in Fig. $4 \mathrm{~b}$ and $4 \mathrm{c}$ represent the outcome of seeding for $t_{\mathrm{sd}}=3 \mathrm{~min}$ and $t_{\mathrm{sd}}=5 \mathrm{~min}$ prior to homogenization (i.e. $\mathrm{t}_{\mathrm{homo}}=0 \mathrm{~min}$ ). A more detailed series is given in Fig. $\mathrm{S} 5$ in the SI. For $\mathrm{t}_{\mathrm{sd}}$ $=3 \mathrm{~min}$ a few $\mathrm{h}-\mathrm{BN}$ domains of very similar sizes have nucleated. At $\mathrm{t}_{\mathrm{sd}}=5 \mathrm{~min}$ these $\mathrm{h}-\mathrm{BN}$ domains have grown in size, but secondary h-BN nucleation has occurred as evidenced by the many additional smaller domains. We note that the onset of secondary nucleation varies and starts occurring from $t_{s d}=3$ min onwards. This highlights a key challenge for controlling the 
microstructure of the resulting h-BN. A high precursor exposure pressure is desirable to minimise incubation time and achieve rapid nucleation and growth (compare to Fig. S6 in SI for $\mathrm{SG}$ at lower $\mathrm{P}_{\mathrm{sd}}$ ), however the accompanying secondary nucleation is detrimental to achieving large domain sizes. We thus introduce a homogenization stage in SSG, and figs. 4b and $4 \mathrm{c}$ show the effects of increasing $\mathrm{t}_{\text {homo. }}$ After $\mathrm{t}_{\mathrm{homo}}=5 \mathrm{~min}$ all smaller h-BN secondary nuclei have disappeared (Fig. 4c) and for $t_{\text {homo }}=10 \mathrm{~min}$ also the larger h-BN domains are removed or significantly reduced in size. Our XPS data (Fig. 2) showed that existing h-BN nuclei are unstable and start to dissociate when borazine is removed. Given a constant rate of dissociation, it is expected that smaller nuclei will disappear first, consistent with our data. This allows us to control the h-BN nucleation density, which justifies our choice $t_{\text {homo }}=5$ min to achieve optimal growth results (Fig. 1a). It should be noted that the overall parameters are highly interdependent. Thus the suggested time of $t_{\text {homo }}=5 \mathrm{~min}$ is optimized for the given set of parameters of temperature, precursor pressure, seeding time and catalyst dimensions. Following homogenization (see Fig. 1a, IV-VII) we then implement a domain growth stage at lower borazine pressure of $2.5 \times 10^{-6} \mathrm{mbar}$. Through this separation of nucleation and domain growth, we can effectively supress secondary nucleation and thus achieve extremely large hBN crystal sizes.

A combination of characterization techniques is used to assess the quality of the h-BN films. Fig. 5a shows a representative bright field (BF) transmission electron microscopy (TEM) image. The h-BN film is only indirectly visible by the presence of a suspended particle (see dotted circle, Fig. 5a), which highlights that the h-BN is uniform and has little contrast and/or features. The selected area electron diffraction pattern in dark field (DF) TEM (Fig. 5b) shows sets of hexagonal diffraction patterns consistent with single crystal mono-layer h-BN. To determine the crystal orientation over a reasonably large area, DF-TEM diffraction patterns 
from various points across the h-BN film were recorded and analysed. We define $\alpha$ as the angle between the vertical and the closest first-order diffraction spot in a clockwise direction (Fig. 5b). The resulting distribution of $\alpha$ is summarised in Figs. 5c and d. For most recorded diffraction patterns $\alpha$ lies within a margin of $\pm 2.5^{\circ}$ of the median $\left(\alpha_{\text {med }}\right)$. The peaks at $-30^{\circ}$ and $30^{\circ}$ correspond to identical orientations due to the hexagonal lattice symmetry. Over the mapped area $(\sim 2 \times 2 \mathrm{~mm})$ the TEM signature is as expected for a single crystal, although we cannot rule out small rotations below the limit of resolution or defects induced by imperfect merging of domains.

These results highlight that the h-BN is highly crystalline and aligned. We relate this result to the epitaxial alignment of h-BN to Pt (llll 11 ). This is shown in greater detail in Fig. S16 of the supplementary information. Low energy electron diffraction (LEED) patterns were recorded of monolayer h-BN grown across $\mathrm{Pt}(111)$ foils grown according to the conditions used for the samples shown in Fig. 1. The six-fold symmetry of the $\operatorname{Pt}(111)$ surface is clearly apparent from the six (01) Pt spots arranged at $60^{\circ}$ from each other. The additional reflexes surrounding the (01) Pt spots are attributable to h-BN, confirming epitaxial alignment between the h-BN lattice and the underlying Pt, with an approximately $9 \times 9$ structure formed due to the mismatch of lattice parameters for h-BN. ${ }^{54-56}$ Measurement of the (01) h-BN spots and (01) Pt spots reveals a ratio of $\sim 1.1$, fully consistent with the $\sim 11 \%$ lattice mismatch between $\mathrm{h}-\mathrm{BN}$ and $\mathrm{Pt}(111)$. We note similar LEED patterns are obtained across a $\sim 5 \times 5 \mathrm{~mm}$ region in the centre of the sample, confirming that the single-crystalline $\operatorname{Pt}(111)$ nature of the foil and that epitaxial alignment of the $\mathrm{h}-\mathrm{BN}$ is achieved across the sample. The h-BN related reflexes show a small angular spread, consistent with the rotational deviations observed by DF-TEM (see Figure 5c,d). As LEED probes an area of hundreds of microns, this is at least partly attributable the comparatively 
higher roughness of our foils with reference to polished bulk-single Pt crystals which have undergone sputter-annealing cycles. ${ }^{54-56}$

Figs. 5e, f show the edge of the film, with only one fringe visible, consistent with mono-layer h-BN. The region marked with the white arrow in Fig. $5 \mathrm{f}$ corresponds to a fold. The contrast between the h-BN mono-layer edge and folded edge can be clearly seen. We further assessed the layer number and homogeneity of as-grown h-BN through SEM and AFM. In all SEM images, where h-BN was immediately imaged, the h-BN domains have homogenous contrast, which indicates a constant number of layers across the whole observed region. AFM measurements show a $\sim 0.4 \mathrm{~nm}$ step height for h-BN after transfer onto $\mathrm{SiO}_{2}$ (see SI, Fig. S10), consistent with mono-layer h-BN thickness.

The SEM images of h-BN flakes shown so far (Figs. 1 and 4), were taken immediately after growth (reactor to SEM transfer time of around 30 min or less). Fig. 6a shows a SEM image recorded 5 hours after removing the sample from the growth chamber, during which time it was stored in ambient environment. Unlike the SEM immediately post-growth of an h-BN domain of similar size and grown under identical conditions (see Fig. 1b V), where the h-BN appears uniformly darker relative to the $\mathrm{Pt}(111)$ substrate, in Fig. 6a the contrast of the h-BN domain is not uniform. The observed lighter edge region (marked "Decoupled" in Fig. 6a) and contrast change is triggered by the intercalation of oxygen/water species. This has been previously observed in SEM and for instance LEEM for various systems of 2DLM on weakly interacting substrates. ${ }^{57-59}$ The change takes place at room temperature within the time scale of hours (see SI, Fig S12), similar to $\mathrm{G} / \mathrm{Pt}^{58}$ and $\mathrm{G} / \mathrm{Cu}{ }^{60}$ The fact that intercalation occurs uniformly from the edges in Figs. 6a and S12 is a further indication of the quality of the h-BN, as the presence of large defects (such as grain boundaries or pinholes) within a h-BN domain 
would reveal itself by the onset of local intercalation. In fact, even for domains that have partially merged, we observe intercalation to only proceed from the edges and not from where any potential GB would be located (see SI, Fig. S13).

The observed intercalation at the interface between h-BN and Pt is an indication of their weak interaction, and a key motivation of our work. Here, we introduce a dry transfer approach to transfer h-BN grown on Pt, as schematically shown in Fig. 6b. A polyvinyl acetate (PVA) stamp is applied to the as-grown h-BN film through drop-casting. The PVA/h-BN stack is then delaminated mechanically (Fig. 6b I). For monolayer h-BN transfer this stack is then stamped down onto the target substrate (Fig. 6b IV) and the stamp is removed by dissolution in water. In line with previous experiments on delamination of $\mathrm{Gr}$ from $\mathrm{Cu},{ }^{16,26}$ we observe an improvement in the ease of transfer by leaving the sample in an ambient environment for an extended period (typically $>24 \mathrm{~h}$ ). We relate this effect to the decoupling of the h-BN layer, consistent with the time dependent change in SE contrast of h-BN on Pt (Fig. 6a; Fig S12 \& $\mathrm{S} 13$ in SI).

The given method of dry transfer is not limited to monolayer transfer but can also be used for the assembly of 2DLM stacks. After picking up the first h-BN layer, the same PVA/h-BN stack can be used for repeated exfoliation of further 2DLM layers. This is performed by simply stamping the stack onto another as-grown 2DLM on its growth substrate (Fig. 6b II) followed by mechanical delamination (Fig. 6b III), which results in the pick-up of an additional 2DLM layer. Such an approach seeks to keep interfacial contamination to a minimum as the second 2DLM layer is only ever in contact with the growth catalyst and the first 2DLM layer. Through sequential pickup in this way, it is possible to assemble stacks of 2DLMs. This approach is not limited to a specific 2DLMs. However, it requires the adhesion between stamp and 2DML to be higher than between 2DLM and substrate, highlighting the need for weakly interacting 
substrates. We use the sequential pick up of 2DLM to fabricate a variety of structures, with Fig. 6c (left) showing an optical image of multilayer h-BN on $\mathrm{SiO}_{2}$ obtained by four sequential peelings of CVD mono-layer h-BN. A heterostructure of CVD h-BN and CVD Gr grown on $\mathrm{Cu}\left(\mathrm{Gr}\right.$ between $\mathrm{h}-\mathrm{BN}$ and $\mathrm{SiO}_{2}$ ) was also fabricated and an optical image of the result is shown in Fig. 6c (right). Since the transfer does not involve any modification of the growth catalyst, the Pt foil can be reused for additional growth cycles. In fact, Pt substrates were used for multiple growth runs (see SI, Fig. S9).

In order to assess the quality of the stacks and to confirm their structure, we employ Raman spectroscopy. The Raman spectrum of h-BN is characterised by the $E_{2 \mathrm{~g}}$ peak at $\sim 1370 \mathrm{~cm}^{-1}$, which due to its non-resonant nature is very weak. ${ }^{61}$ However, its peak position and relative intensity can offer an insight into the thickness of the h-BN. ${ }^{61-63}$ Fig. $6 \mathrm{~d}$ shows such Raman characterization of multilayer h-BN obtained through repeated exfoliation. The Raman spectra given in Fig. 6d (left) show an increase in $\mathrm{E}_{2 \mathrm{~g}}$ peak area, which is proportional to the number of sequential transfers. This demonstrates that using our transfer method we are able to pickup an additional layer during each cycle. Furthermore, it should be noted that the full-width at half maximum (FWHM) of the $\mathrm{E}_{2 \mathrm{~g}}$ peak of monolayer h-BN is $\sim 13 \mathrm{~cm}^{-1}$, which is comparable to exfoliated h-BN. ${ }^{61}$ Since the FWHM is often cited as an indicator of material quality, it highlights the high quality of our CVD h-BN. ${ }^{63}$ Fig. $6 \mathrm{~d}$ (right) reflects the results of Raman mapping across $100 \mu \mathrm{m} \times 100 \mu \mathrm{m}$, whereby the quantitative analysis is based on fitting Lorentzian curves to the $\mathrm{E}_{2 \mathrm{~g}}$ peak. The linear relationship between peak area and layer number is maintained across the mapped region, indicating the quality of the transfer method. Furthermore, in agreement with previous studies, ${ }^{62}$ we observe a slight red-shift in the $\mathrm{E}_{2 \mathrm{~g}}$ peak position with increasing layer number from $1369.7 \mathrm{~cm}^{-1}$ for monolayer h-BN to $1368.7 \mathrm{~cm}^{-1}$ for four-layer h-BN, which indicates a clean interface as this shift relates to the interaction between 
layers. ${ }^{61,62}$ The Raman characterization of $\mathrm{h}-\mathrm{BN} / \mathrm{Gr}$ heterostructures is more challenging, due to the large difference in signal intensity of Gr and h-BN. The Raman spectrum of an all CVD heterostructure is shown in Fig. 6e. Due to the proximity of peaks, minor imperfections in the Gr will result in the Gr D-peak $\left(1350 \mathrm{~cm}^{-1}\right)^{64}$ overlapping with and potentially swamping the hBN peak $\left(1370 \mathrm{~cm}^{-1}\right)$. In Fig. 6e the Gr D-peak/G-peak ratio is less than 0.025, still the h-BN peak is only just visible in the magnification of the plot (inset), demonstrating that Gr can be directly delaminated from the growth catalyst using h-BN.

The fabrication of high quality $\mathrm{Gr} / \mathrm{h}-\mathrm{BN}$ heterostructures has been a significant challenge even when relying entirely on exfoliation..$^{24,65}$ Thus, to demonstrate the feasibility of our approach and to narrow-down the parameter space for device processing, we focus here on just the insertion of a CVD h-BN layer via a model structure consisting of monolayer CVD-h-BN as a capping layer on monolayer exfoliated (exf) Gr. Fig. 7a shows an optical image of the assembled stack. Using a PVA/CVD h-BN stack, which we obtain by delaminating an as grown h-BN layer from Pt, we pick up exf-Gr from a $\mathrm{SiO}_{2} / \mathrm{Si}$ wafer. The h-BN is not observed optically as it uniformly covers the sample. It becomes apparent in Fig. 7a II and III, which presents the result of a peak force (PF-) AFM measurement of the transition region from CVD h-BN/exf-Gr to CVD h-BN only. The measured step height for the exf-Gr layer is $\sim 0.4 \mathrm{~nm}$ and the surface is atomically smooth, which are indications of an interface without significant amounts of trapped residues. Fig. $7 b$ shows the result of the Raman analysis. The Gr G peak and $2 \mathrm{D}$ peak position of each spectrum is plotted with the strain and doping axis for reference. ${ }^{66}$ The colour of each point indicates the FWHM of the Gr 2D peak. The median of the Gr G peak and 2D peak positions are $1584.7 \mathrm{~cm}^{-1}$ and $2684.6 \mathrm{~cm}^{-1}$ respectively, compared to $1581.6 \mathrm{~cm}^{-}$ ${ }^{1}$ and $2676.9 \mathrm{~cm}^{-1}$ for suspended exf-Gr. ${ }^{66}$ The median FWHM of the Gr 2D peak is $26.2 \mathrm{~cm}^{-1}$, which is similar to previous studies, where exf-h-BN/exf-Gr on $\mathrm{SiO}_{2}$ has a 2D peak FWHM of 
about $25 \mathrm{~cm}^{-1} \cdot{ }^{67}$ The measurement points are aligned along the strain axis, indicating that the sample is undoped, but is affected by strain.

The sheet conductivity of a representative CVD h-BN/exf-Gr field effect transistor (FET) device (where the Si substrate acts a back gate) is shown in Fig. 7c. The position of the charge neutrality point (CNP), at $-0.2 \mathrm{~V}$, confirms that the graphene is highly intrinsic. The devices are shaped as Hall bars, allowing independent measurement of gate-dependent conductivity and charge carrier concentration (by applying an out of plane magnetic field). The measurements were carried out under vacuum conditions. Further details are given in the Experimental Section. The Hall mobility $\left(\mu_{\mathrm{H}}\right)$ is then extracted assuming a Drude model for conductivity, leading to a peak $\mu_{\mathrm{H}}=7200 \mathrm{~cm}^{2} \mathrm{~V}^{-1} \mathrm{~s}^{-1}$ at room temperature. The charge carrier density without the application of a back-gate voltage is $\mathrm{n}_{0}=4.8 \times 10^{10} \mathrm{~cm}^{-2}$, confirming the low doping level indicated by the Raman analysis. We note that such low doping is achieved without the need for any high temperature annealing to remove residuals post-transfer. We repeated the analysis for three independent devices and find the values reproducible. The performance of the given devices underscores the cleanliness of the graphene/h-BN interface and is, to the best of our knowledge, the highest for heterostructures using CVD h-BN. Further improvements in mobility should be achievable by introducing an additional h-BN layer underneath the Gr to screen the roughness and charged impurities of the $\mathrm{SiO}_{2}{ }^{65}$ The given stack serves as a first demonstrator for our proposed approach of growing h-BN via CVD and integrating it into heterostructure devices with the goal to replace exfoliated h-BN. Beyond such devices, the ability to grow $\mathrm{h}-\mathrm{BN}$ in a fast process that allows for clean device integration is relevant for a variety of other applications such as single photon emitters, ${ }^{68}$ nano-resonators ${ }^{69}$ and magnetic tunnel junctions. ${ }^{70}$ 


\section{Conclusions}

Improving current 2DLM integration strategies requires careful consideration of the growth catalyst. Here, we demonstrate that Pt is a suitable weakly interacting catalyst that enables not only h-BN growth with mono-layer control and crystal sizes in excess of $0.5 \mathrm{~mm}$, but also direct transfer by delaminating as-grown layers to achieve clean processing. We establish that h-BN grows isothermally on Pt within the given CVD parameter space, driven by a local B and $\mathrm{N}$ supersaturation at the surface, and that when the precursor flux is removed the h-BN layer starts to dissolve. We find that initial B dissolution and decoration of Pt GBs leads to significantly accelerated Pt crystallisation. These insights allowed us to devise an integrated CVD growth process, that we referred to as SSG, which based on two coupled borazine exposures not only transforms poly-crystalline Pt foils into a dominant (111) orientation but enables independent control of h-BN nucleation and domain expansion, i.e. the CVD of highly crystalline mono-layer h-BN. The SSG process enables growth times of less than 45 min for these large h-BN crystal sizes and a merged, continuous layer. This compares favourably to the latest literature, which reports h-BN grain sizes of $\sim 15 \mu \mathrm{m}$ after a growth time of $50 \mathrm{~min}$, and full coverage at beyond $1 \mathrm{~h}$ on liquid Au. ${ }^{71}$ Importantly, in contrast to most such previous literature that uses wet transfer/electrochemical delamination, we here have demonstrated a delamination transfer method that makes use of the weak $\mathrm{Pt} / \mathrm{h}-\mathrm{BN}$ interaction, to directly delaminate the as-grown films from the catalyst. We thereby preserve the catalyst for regrowth and can achieve a clean transfer process. This approach allows the precise control of the thickness of the h-BN layer, something that has not been achieved for exfoliated h-BN, but is critical to many applications. Scalable manufacture, especially of clean 2DLM heterostructures, has been a main bottleneck for the whole field. While further process 
optimization is still required, our study demonstrates an approach towards achieving this elusive goal. 


\section{Experimental Methods}

\section{h-BN Growth}

All samples, except for in situ XPS experiments (Fig. 2), are grown in a custom-built laser CVD reactor. An $808 \mathrm{~nm}$ continuous wave (CW) IR Laser with a maximum power of $60 \mathrm{~W}$ is used for heating. It is positioned outside of the CVD chamber. The light is coupled into the chamber through a laser window. In order to achieve a homogeneous beam profile, a beam shaper is used that creates a top-hat (instead of Gaussian) profile at the focal point, which has a size of $5 \mathrm{~mm}$ x $5 \mathrm{~mm}$. Due to very localized heating, only the sample itself is significantly heated, thus the system under discussion falls in the category of cold-wall reactors. We measure the temperature using an IR pyrometer with a wavelength of $1.6 \mu \mathrm{m}$, a focal spot diameter of $3 \mathrm{~mm}$ and the emissivity is set at $0.25 .^{72}$ The estimated uncertainty is $\pm 50{ }^{\circ} \mathrm{C}$.

If not specified otherwise, the as received Pt foil without pre-treatment $(25 \mu \mathrm{m}, 99.99 \%$, Alfa Aesar) is mounted on a tantalum (Ta) foil $(25 \mu \mathrm{m}, 99.9 \%$, Goodfellow), which acts as the susceptor, enabling homogenous heating across a larger area than the size of the beam at the focal point. The Ta susceptor is clamped using sapphire in a custom-made mounting stage, and the Pt foil does not come into contact with anything but the susceptor, thus avoiding contamination and thermal dissipation. After loading the sample into the chamber, it is pumped down to achieve a base pressure of less than $2 \times 10^{-6}$ mbar before starting to heat to the growth temperature under vaccuum. The pressure is measured using a full range pressure gauge consisting of Pirani and cold cathode gauges for different pressure regimes. H-BN growth is performed by introducing borazine ( $>97 \%$, Fluorochem) into the chamber with a manual leak valve with the sample held at a fixed temperature. The borazine is stored at room temperature. The exact procedure to obtain large h-BN domains is detailed in Fig. 1 and applies also to 
repeated use of the same Pt substrate. Other gases that have been used in reference experiments, e.g. to investigate the effect of the atmosphere on the crystallinity of the Pt substrate (see Fig. 3) are injected into the chamber using mass flow controllers.

\section{Transfer}

Peeling transfer As the carrier layer for exfoliating the h-BN post-growth, we use a solution of 5g PVA (Mw 9000-10000, 80\% hydrolized, Sigma Aldrich) and 1g glycerol as a softener (>99\%, Sigma Aldrich) in $100 \mathrm{ml}$ of de-ionized (DI) water. In the first step, we drop-cast the solution on the sample and dry at $80^{\circ} \mathrm{C}$ for $20 \mathrm{~min}$. Then, the $s t a m p / h-B N$ film is peeled off using tweezers, stamped onto the target substrate at $125^{\circ} \mathrm{C}$, and annealed for $5 \mathrm{~min}$. In the case of multilayer h-BN or h-BN/graphene heterostructures, the target substrate is another CVD grown 2DLM sample and the peeling process is repeated. In the final step, the stamp with the 2DLM layer is put down onto the substrate of choice and the carrier layer is dissolved in DI water at $50^{\circ} \mathrm{C}$ for at least $3 \mathrm{~h}$.

Bubbling transfer The samples are spin-coated with Poly(methyl methacrylate) (PMMA, $495 \mathrm{k}$ ) at $3000 \mathrm{rpm}$ for $40 \mathrm{~s}$ then baked at $180^{\circ} \mathrm{C}$ for 1.5 min During the actual transfer process, $\mathrm{NaOH}$ solution (1M, a.q.) is used as electrolyte, and the sample (h-BN coated with PMMA) is used as the negative electrode and a Pt wire as the positive electrode. The typical settings are $\sim 4.5 \mathrm{~V}$ and $\sim 0.3 \mathrm{~A}$. PMMA supported h-BN fully delaminates from Pt within 5 minutes. The sample is then rinsed in DI water three times, with each rinse lasting $\sim 45$ minutes, and then transferred onto the desired substrate. 


\section{Characterization}

Raman measurements were performed with a Renishaw inVia confocal Raman Microscope. $514 \mathrm{~nm}$ or $532 \mathrm{~nm}$ lasers were used depending on equipment availability. Spectra were taken with a 50x objective lens. A step size of $2 \mu \mathrm{m}$ was used for all maps. All Raman spectra are taken after transfer onto $\mathrm{SiO}_{2} / \mathrm{Si}$ substrates. The SEM images were taken in the FEI Magellan SEM using an acceleration voltage of $1 \mathrm{kV}$. The TEM images (FEI Osiris TEM) were taken with an acceleration voltage of $40 \mathrm{kV}$. The knock-on damage of h-BN is minimized under such a low acceleration voltage. The samples are transferred using bubbling transfer in this case, as direct peeling requires PVA dissolution in DI water to release the h-BN film and the water's high surface tension is likely to damage the suspended h-BN. For the bubbling transfer only low surface tension solvents are used to release the h-BN.

In situ XPS measurements were performed at the BESSY II synchrotron at the ISISS end station of the FHI-MPG. The setup consists of a reaction cell (base pressure $\approx 10^{-8}$ mbar) attached to an analyser with a differentially pumped electrostatic lens system (Phoibos 150 NAP, SPECS GmbH). ${ }^{73} \mathrm{XP}$ core level spectra were collected in normal emission geometry using a x-ray beam with a spot size of $\sim 80 \mu \mathrm{m} \times 150 \mu \mathrm{m}$. All spectra are background-corrected (linear) and their binding energies are referenced to the contemporaneously measured Fermi edges. The temperature is measured using a dual-wavelength pyrometer.

AFM was measured in peak force tapping mode using a Bruker Dimension Icon AFM. In this mode, the feedback loop keeps the peak force of tip-sample interaction constant.

XRD was carried out on a Philips X'pert MRD diffractometer with a $\mathrm{Cu} \mathrm{K}_{\alpha 1} \mathrm{X}$-ray source ( $\lambda$ $=1.5405974 \AA$ ) and a 4-bounce $\mathrm{Ge}(220)$ asymmetric monochromator. The spot size is $5 \mathrm{~mm}$ x $15 \mathrm{~mm}$. 


\section{Heterostructure assembly, Device Fabrication \& Measurement}

Continuous monolayer CVD h-BN on Pt was picked up/delaminated from Pt using the peeling procedure described above. The sample is then used to pick up Gr, which was prepared in advance by mechanical exfoliation from a bulk crystal onto a $\mathrm{SiO}_{2} / \mathrm{Si}$ wafer. For heterostructure assembly, the PVA/h-BN layer is pressed onto the wafer with $\mathrm{Gr}$ at $30^{\circ} \mathrm{C}$ and then peeled off. This stack is then stamped onto another wafer at a temperature of $130^{\circ} \mathrm{C}$. The sample is heated at $130{ }^{\circ} \mathrm{C}$ for $5 \mathrm{~min}$, before dissolving the PVA film in DI water.

In order to probe the electronic transport properties of the heterostructure we fabricate fourterminal transport geometries following an established method. ${ }^{23,25}$ We begin by deposition of an aluminium $(\mathrm{Al})$ etch mask fabricated by electron-beam (e-beam) lithography, followed by thermal evaporation of $30 \mathrm{~nm}$ of $\mathrm{Al}$, and lift-off. Following this, the exposed regions of $\mathrm{Gr} / \mathrm{h}$ $\mathrm{BN}$ are etched with a reactive ion etcher (RIE) using $\mathrm{CF}_{4}$ gas under a forward RF power of 20W. The Al mask is then removed by wet etching. Finally, metal contact leads are deposited by patterning with e-beam lithography followed by DC sputtering of $5 / 70 \mathrm{~nm}$ of $\mathrm{Cr} / \mathrm{Cu}$ and liftoff.

Four-terminal transport measurements are performed in a Lakeshore Cryogenic probe station at a pressure of $\sim 4 \times 10^{-8}$ Torr and a temperature $\mathrm{T}=290 \mathrm{~K}$. The resistance is measured using a dual lock-in amplifier set-up at a frequency of $\sim 13 \mathrm{~Hz}$ and bias current $\sim 100 \mathrm{nA}$. The gate voltage is swept using an SMU. 


\section{References}

(1) Ten Years in Two Dimensions. Ten years two Dimens. Nat. Nanotechnol. [Special Issue] 2014, 9.

(2) Hofmann, S.; Braeuninger-Weimer, P.; Weatherup, R. S. CVD-Enabled Graphene Manufacture and Technology. J. Phys. Chem. Lett. 2015, 6, 2714-2721.

(3) Pakdel, A.; Bando, Y.; Golberg, D. Nano Boron Nitride Flatland. Chem. Soc. Rev. 2014, 43, 934-959.

(4) Caneva, S.; Weatherup, R. S.; Bayer, B. C.; Blume, R.; Cabrero-Vilatela, A.; Braeuninger-Weimer, P.; Martin, M.-B.; Wang, R.; Baehtz, C.; Schloegl, R.; Meyer, J. C.; Hofmann, S. Controlling Catalyst Bulk Reservoir Effects for Monolayer Hexagonal Boron Nitride CVD. Nano Lett. 2016, 16, 1250-1261.

(5) Loginova, E.; Bartelt, N. C.; Feibelman, P. J.; McCarty, K. F. Factors Influencing Graphene Growth on Metal Surfaces. New J. Phys. 2009, 11, 63046.

(6) Bartelt, N. C.; McCarty, K. F. Graphene Growth on Metal Surfaces. MRS Bull. 2012, $37,1158-1165$.

(7) Kidambi, P. R.; Blume, R.; Kling, J.; Wagner, J. B.; Baehtz, C.; Weatherup, R. S.; Schloegl, R.; Bayer, B. C.; Hofmann, S. In Situ Observations during Chemical Vapor Deposition of Hexagonal Boron Nitride on Polycrystalline Copper. Chem. Mater. 2014, $26,6380-6392$.

(8) Wu, T.; Zhang, X.; Yuan, Q.; Xue, J.; Lu, G.; Liu, Z.; Wang, H.; Wang, H.; Ding, F.; Yu, Q. Fast Growth of Inch-Sized Single-Crystalline Graphene from a Controlled Single Nucleus on Cu-Ni Alloys. Nat. Mater. 2016, 15, 43-47. 
(9) Braeuninger-Weimer, P.; Brennan, B.; Pollard, A. J.; Hofmann, S. Understanding and Controlling Cu-Catalyzed Graphene Nucleation: The Role of Impurities, Roughness, and Oxygen Scavenging. Chem. Mater. 2016, 28, 8905-8915.

(10) Lee, J.-H.; Lee, E. K.; Joo, W.-J.; Jang, Y.; Kim, B.-S.; Lim, J. Y.; Choi, S.-H.; Ahn, S. J.; Ahn, J. R.; Park, M.-H.; Yang, C.-W.; Choi, B. L.; Hwang, S.-W.; Whang, D. WaferScale Growth of Single-Crystal Monolayer Graphene on Reusable HydrogenTerminated Germanium. Science 2014, 344, 286-289.

(11) Xu, X.; Zhang, Z.; Dong, J.; Yi, D.; Niu, J.; Wu, M.; Lin, L.; Yin, R.; Li, M.; Zhou, J.; Wang, S.; Sun, J.; Duan, X.; Gao, P.; Jiang, Y.; Wu, X.; Peng, H.; Ruoff, R. S.; Liu, Z.; Yu, D.; et al. Ultrafast Epitaxial Growth of Metre-Sized Single-Crystal Graphene on Industrial Cu Foil. Sci. Bull. 2017, 62, 1074-1080.

(12) Li, X. S.; Cai, W. W.; An, J. H.; Kim, S.; Nah, J.; Yang, D. X.; Piner, R.; Velamakanni, A.; Jung, I.; Tutuc, E.; Banerjee, S. K.; Colombo, L.; Ruoff, R. S. Large-Area Synthesis of High-Quality and Uniform Graphene Films on Copper Foils. Science 2009, 324, $1312-1314$.

(13) Kim, K. S.; Zhao, Y.; Jang, H.; Lee, S. Y.; Kim, J. M.; Kim, K. S.; Ahn, J.-H.; Kim, P.; Choi, J.-Y.; Hong, B. H. Large-Scale Pattern Growth of Graphene Films for Stretchable Transparent Electrodes. Nature 2009, 457, 706-710.

(14) Yoon, T.; Shin, W. C.; Kim, T. Y.; Mun, J. H.; Kim, T.-S.; Cho, B. J. Direct Measurement of Adhesion Energy of Monolayer Graphene As-Grown on Copper and Its Application to Renewable Transfer Process. Nano Lett. 2012, 12, 1448-1452.

(15) Bae, S.-H.; Zhou, X.; Kim, S.; Lee, Y. S.; Cruz, S. S.; Kim, Y.; Hannon, J. B.; Yang, Y.; Sadana, D. K.; Ross, F. M. Unveiling the Carrier Transport Mechanism in Epitaxial 
Graphene for Forming Wafer-Scale, Single-Domain Graphene. Proc. Natl. Acad. Sci. 2017, 114, 4082-4086.

(16) Whelan, P. R.; Jessen, B. S.; Wang, R.; Luo, B.; Stoot, A. C.; Mackenzie, D. M. A.; Braeuninger-Weimer, P.; Jouvray, A.; Prager, L.; Camilli, L. Raman Spectral Indicators of Catalyst Decoupling for Transfer of CVD Grown 2D Materials. Carbon N. Y. 2017, $117,75-81$.

(17) Wang, Y.; Zheng, Y.; Xu, X.; Dubuisson, E.; Bao, Q.; Lu, J.; Loh, K. P. Electrochemical Delamination of CVD-Grown Graphene Film: Toward the Recyclable Use of Copper Catalyst. ACS Nano 2011, 5, 9927-9933.

(18) Liu, L.; Liu, X.; Zhan, Z.; Guo, W.; Xu, C.; Deng, J.; Chakarov, D.; Hyldgaard, P.; Schröder, E.; Yurgens, A. A Mechanism for Highly Efficient Electrochemical Bubbling Delamination of CVD-Grown Graphene from Metal Substrates. Adv. Mater. Interfaces 2015, 3, 150049 .

(19) Verguts, K.; Schouteden, K.; Wu, C. H.; Peters, L.; Vrancken, N.; Wu, X.; Li, Z.; Erkens, M.; Porret, C.; Huyghebaert, C.; Van Haesendonck, C.; De Gendt, S.; Brems, S. Controlling Water Intercalation Is Key to a Direct Graphene Transfer. ACS Appl. Mater. Interfaces 2017, 9, 37484-37492.

(20) Wang, R.; Whelan, P. R.; Braeuninger-Weimer, P.; Tappertzhofen, S.; AlexanderWebber, J. A.; Van-Veldhoven, Z. A.; Kidambi, P. R.; Jessen, B. S.; Booth, T. J.; Boggild, P. Catalyst Interface Engineering for Improved 2D Film Lift-off and Transfer. ACS Appl. Mater. Interfaces 2016, 8, 33072-33082.

(21) Pirkle, A.; Chan, J.; Venugopal, A.; Hinojos, D.; Magnuson, C. W.; McDonnell, S.; Colombo, L.; Vogel, E. M.; Ruoff, R. S.; Wallace, R. M. The Effect of Chemical 
Residues on the Physical and Electrical Properties of Chemical Vapor Deposited Graphene Transferred to SiO2. Appl. Phys. Lett. 2011, 99, 122108.

(22) Lupina, G.; Kitzmann, J.; Costina, I.; Lukosius, M.; Wenger, C.; Wolff, A.; Vaziri, S.; Östling, M.; Pasternak, I.; Krajewska, A.; Strupinski, W.; Kataria, S.; Gahoi, A.; Lemme, M. C.; Ruhl, G.; Zoth, G.; Luxenhofer, O.; Mehr, W. Residual Metallic Contamination of Transferred Chemical Vapor Deposited Graphene. ACS Nano 2015, $9,4776-4785$.

(23) Wang, L.; Meric, I.; Huang, P. Y.; Gao, Q.; Gao, Y.; Tran, H.; Taniguchi, T.; Watanabe, K.; Campos, L. M.; Muller, D. A.; Guo, J.; Kim, P.; Hone, J.; Shepard, K. L.; Dean, C. R. One-Dimensional Electrical Contact to a Two-Dimensional Material. Science 2013, $342,614-617$.

(24) Pizzocchero, F.; Gammelgaard, L.; Jessen, B. S.; Caridad, J. M.; Wang, L.; Hone, J.; Bøggild, P.; Booth, T. J. The Hot Pick-up Technique for Batch Assembly of van Der Waals Heterostructures. Nat. Commun. 2016, 7, 11894.

(25) Purdie, D. G.; Pugno, N. M.; Taniguchi, T.; Watanabe, K.; Ferrari, A. C.; Lombardo, A. Cleaning Interfaces in Layered Materials Heterostructures. Nat. Commun. 2018, 9, 5387.

(26) Banszerus, L.; Schmitz, M.; Engels, S.; Dauber, J.; Oellers, M.; Haupt, F.; Watanabe, K.; Taniguchi, T.; Beschoten, B.; Stampfer, C. Ultrahigh-Mobility Graphene Devices from Chemical Vapor Deposition on Reusable Copper. Sci. Adv. 2015, 1, e1500222.

(27) Banszerus, L.; Schmitz, M.; Engels, S.; Goldsche, M.; Watanabe, K.; Taniguchi, T.; Beschoten, B.; Stampfer, C. Ballistic Transport Exceeding $28 \mathrm{Mm}$ in CVD Grown Graphene. Nano Lett. 2016, 16, 1387-1391. 
(28) Hayne, W. Melting, Boiling, Triple, and Critical Points of the Elements; CRC Press, 2016.

(29) Gao, Y.; Ren, W.; Ma, T.; Liu, Z.; Zhang, Y.; Liu, W.-B.; Ma, L.-P.; Ma, X.; Cheng, H.-M. Repeated and Controlled Growth of Monolayer, Bilayer and Few-Layer Hexagonal Boron Nitride on Pt Foils. ACS Nano 2013, 7, 5199-5206.

(30) Park, J.-H.; Park, J. C.; Yun, S. J.; Kim, H.; Luong, D. H.; Kim, S. M.; Choi, S. H.; Yang, W.; Kong, J.; Kim, K. K.; Lee, Y. H. Large-Area Monolayer Hexagonal Boron Nitride on Pt Foil. ACS Nano 2014, 8, 8520-8528.

(31) Paffett, M. T.; Simonson, R. J.; Papin, P.; Paine, R. T. Borazine Adsorption and Decomposition at $\mathrm{Pt}(111)$ and $\mathrm{Ru}(001)$ Surfaces. Surf. Sci. 1990, 232, 286-296.

(32) Preobrajenski, A. B.; Nesterov, M. A.; Ng, M. L.; Vinogradov, A. S.; Mårtensson, N. Monolayer H-BN on Lattice-Mismatched Metal Surfaces: On the Formation of the Nanomesh. Chem. Phys. Lett. 2007, 446, 119-123.

(33) Kilday, M. V; Johnson, W. H.; Pros, E. J. Heat of Combustion of Borazine. J. Res. Natl. Bur. Stand. (1934). 1961, 65, 101-104.

(34) Babenko, V.; Lane, G.; Koos, A. A.; Murdock, A. T.; So, K.; Britton, J.; Meysami, S. S.; Moffat, J.; Grobert, N. Time Dependent Decomposition of Ammonia Borane for the Controlled Production of 2D Hexagonal Boron Nitride. Sci. Rep. 2017, 7, 1-12.

(35) Trehan, R.; Lifshitz, Y.; Rabalais, J. W. Auger and X-Ray Electron Spectroscopy Studies of h-BN, c-BN, and N2 Ion Irradiation of Boron and Boron Nitride. J. Vac. Sci. Technol. A Vacuum, Surfaces, Film. 1990, 8, 4026-4032.

(36) Preobrajenski, A. B.; Vinogradov, A. S.; Mårtensson, N. Monolayer of H-BN 
Chemisorbed on $\mathrm{Cu}\left(\begin{array}{lll}1 & 1 & 1\end{array}\right)$ and $\mathrm{Ni}\left(\begin{array}{lll}1 & 1 & 1\end{array}\right)$ : The Role of the Transition Metal 3d States. Surf. Sci. 2005, 582, 21-30.

(37) Ma, T.; Ren, W.; Liu, Z.; Huang, L.; Ma, L. P.; Ma, X.; Zhang, Z.; Peng, L. M.; Cheng, H. M. Repeated Growth-Etching-Regrowth for Large-Area Defect-Free Single-Crystal Graphene by Chemical Vapor Deposition. ACS Nano 2014, 8, 12806-12813.

(38) Li, L. H.; Cervenka, J.; Watanabe, K.; Taniguchi, T.; Chen, Y. Strong Oxidation Resistance of Atomically Thin Boron Nitride Nanosheets. ACS Nano 2014, 8, 14571462.

(39) Liu, Z.; Gong, Y.; Zhou, W.; Ma, L.; Yu, J.; Idrobo, J. C.; Jung, J.; MacDonald, A. H.; Vajtai, R.; Lou, J. Ultrathin High-Temperature Oxidation-Resistant Coatings of Hexagonal Boron Nitride. Nat. Commun. 2013, 4, 2541.

(40) Predel, B. B-Pt (Boron-Platinum). In Landolt-Börnstein - Group IV Physical Chemistry; Madelung, O., Ed.; Springer-Verlag Berlin Heidelberg, 1992; Vol. 5B.

(41) Weatherup, R. S.; Dlubak, B.; Hofmann, S. Kinetic Control of Catalytic CVD for HighQuality Graphene at Low Temperatures. ACS Nano 2012, 6, 9996-10003.

(42) Tanuma, S.; Powell, C. J.; Penn, D. R. Calculations of Electron Inelastic Mean Free Paths. IX. Data for 41 Elemental Solids over the $50 \mathrm{EV}$ to $30 \mathrm{KeV}$ Range. Surf. Interface Anal. 2011, 43, 689-713.

(43) Weatherup, R. S.; Shahani, A. J.; Wang, Z.-J.; Mingard, K.; Pollard, A. J.; Willinger, M.-G.; Schloegl, R.; Voorhees, P. W.; Hofmann, S. In Situ Graphene Growth Dynamics on Polycrystalline Catalyst Foils. Nano Lett. 2016, 16, 6196-6206.

(44) Brown, L.; Lochocki, E. B.; Avila, J.; Kim, C. J.; Ogawa, Y.; Havener, R. W.; Kim, D. 
K.; Monkman, E. J.; Shai, D. E.; Wei, H. I.; Levendorf, M. P.; Asensio, M.; Shen, K. M.; Park, J. Polycrystalline Graphene with Single Crystalline Electronic Structure. Nano Lett. 2014, 14, 5706-5711.

(45) Nguyen, V. L.; Shin, B. G.; Duong, D. L.; Kim, S. T.; Perello, D.; Lim, Y. J.; Yuan, Q. H.; Ding, F.; Jeong, H. Y.; Shin, H. S. Seamless Stitching of Graphene Domains on Polished Copper (111) Foil. Adv. Mater. 2015, 27, 1376-1382.

(46) Thompson, C. V. Grain Growth in Thin Films. Annu. Rev. Mater. Sci. 1990, 20, 245268.

(47) Lee, D. S.; Park, D. Y.; Woo, H. J.; Kim, S. H.; Ha, J.; Yoon, E. Preferred Orientation Controlled Giant Grain Growth of Platinum Thin Films on SiO 2/Si Substrates. Japanese J. Appl. Physics, Part 2 Lett. 2001, 40, 10-13.

(48) Gladman, T. Grain Size Control; OCP science Philadelphia, Pa/USA, 2004.

(49) Humphreys, F. J.; Hatherly, M. Recrystallization and Related Annealing Phenomena; Elsevier, 2012.

(50) Beck, G.; Bachmann, C. Oxygen Removal at Grain Boundaries in Platinum Films on YSZ. Solid State Ionics 2014, 262, 508-511.

(51) Kim, M. H.; Park, T.-S.; Yoon, E.; Lee, D.-S.; Park, D.-Y.; Woo, H.-J.; Chun, D.-I.; Ha, J. Changes in Preferred Orientation of Pt Thin Films Deposited by DC Magnetron Sputtering Using Ar/O2 Gas Mixtures. J. Mater. Res. 1999, 14, 1255-1260.

(52) Preußner, J.; Fleischmann, E.; Völk1, R.; Glatzel, U. Enrichment of Boron at Grain Boundaries of Platinum-Based Alloys Determined by Electron Energy Loss Spectroscopy in a Transmission Electron Microscope. Int. J. Mater. Res. 2010, 101, 
$577-579$.

(53) Davis, J. R. Copper and Copper Alloys; ASM international, 2001.

(54) Müller, F.; Stöwe, K.; Sachdev, H. Symmetry versus Commensurability: Epitaxial Growth of Hexagonal Boron Nitride on Pt(111) from B-Trichloroborazine (ClBNH)3. Chem. Mater. 2005, 17, 3464-3467.

(55) Preobrajenski, A. B.; Vinogradov, A. S.; Ng, M. L.; Ćavar, E.; Westerström, R.; Mikkelsen, A.; Lundgren, E.; Mårtensson, N. Influence of Chemical Interaction at the Lattice-Mismatched Interfaces on the Overlayer Morphology. Phys. Rev. B 2007, 75, 245412.

(56) Ćavar, E.; Westerström, R.; Mikkelsen, A.; Lundgren, E.; Vinogradov, A. S.; Ng, M. L.; Preobrajenski, A. B.; Mårtensson, N.; Vinogradov, S. A Single H-BN Layer on Pt (1 1 1). Surf. Sci. 2008, 602, 1722-1726.

(57) Yao, Y.; Fu, Q.; Zhang, Y. Y.; Weng, X.; Li, H.; Chen, M.; Jin, L.; Dong, A.; Mu, R.; Jiang, P. Graphene Cover-Promoted Metal-Catalyzed Reactions. Proc. Natl. Acad. Sci. 2014, 111, 17023-17028.

(58) Weatherup, R. S.; D’Arsié, L.; Cabrero-Vilatela, A.; Caneva, S.; Blume, R.; Robertson, J.; Schloegl, R.; Hofmann, S. Long-Term Passivation of Strongly Interacting Metals with Single-Layer Graphene. J. Am. Chem. Soc. 2015, 137, 14358-14366.

(59) Ng, M. L.; Shavorskiy, A.; Rameshan, C.; Mikkelsen, A.; Lundgren, E.; Preobrajenski, A.; Bluhm, H. Reversible Modification of the Structural and Electronic Properties of a Boron Nitride Monolayer by CO Intercalation. ChemPhysChem 2015, 16, 923-927.

(60) Blume, R.; Kidambi, P. R.; Bayer, B. C.; Weatherup, R. S.; Wang, Z.-J.; Weinberg, G.; 
Willinger, M.-G.; Greiner, M.; Hofmann, S.; Knop-Gericke, A.; Schlogl, R. The Influence of Intercalated Oxygen on the Properties of Graphene on Polycrystalline $\mathrm{Cu}$ under Various Environmental Conditions. Phys. Chem. Chem. Phys. 2014, 16, 2598926003.

(61) Gorbachev, R. V; Riaz, I.; Nair, R. R.; Jalil, R.; Britnell, L.; Belle, B. D.; Hill, E. W.; Novoselov, K. S.; Watanabe, K.; Taniguchi, T. Hunting for Monolayer Boron Nitride: Optical and Raman Signatures. Small 2011, 7, 465-468.

(62) Cai, Q.; Scullion, D.; Falin, A.; Watanabe, K.; Taniguchi, T.; Chen, Y.; Santos, E. J. G.; Li, L. H. Raman Signature and Phonon Dispersion of Atomically Thin Boron Nitride. Nanoscale 2017, 9, 3059-3067.

(63) Schué, L.; Stenger, I.; Fossard, F.; Loiseau, A.; Barjon, J. Characterization Methods Dedicated to Nanometer-Thick HBN Layers. 2D Mater. 2017, 4, 015028.

(64) Ferrari, A. C. Raman Spectroscopy of Graphene and Graphite: Disorder, ElectronPhonon Coupling, Doping and Nonadiabatic Effects. Solid State Commun. 2007, 143, $47-57$.

(65) Dean, C. R.; Young, A. F.; Meric, I.; Lee, C.; Wang, L.; Sorgenfrei, S.; Watanabe, K.; Taniguchi, T.; Kim, P.; Shepard, K. L.; Hone, J. Boron Nitride Substrates for HighQuality Graphene Electronics. Nat. Nanotechnol. 2010, 5, 722-726.

(66) Lee, J. E.; Ahn, G.; Shim, J.; Lee, Y. S.; Ryu, S. Optical Separation of Mechanical Strain from Charge Doping in Graphene. Nat. Commun. 2012, 3, 1024-1028.

(67) Neumann, C.; Reichardt, S.; Venezuela, P.; Drogeler, M.; Banszerus, L.; Schmitz, M.; Watanabe, K.; Taniguchi, T.; Mauri, F.; Beschoten, B.; Rotkin, S. V; Stampfer, C. 
Raman Spectroscopy as Probe of Nanometre-Scale Strain Variations in Graphene. Nat. Commun. 2015, 6, 8429.

(68) Tran, T. T.; Bray, K.; Ford, M. J.; Toth, M.; Aharonovich, I. Quantum Emission from Hexagonal Boron Nitride Monolayers. Nat. Nanotechnol. 2016, 11, 37-41.

(69) Cartamil-Bueno, S. J.; Cavalieri, M.; Wang, R.; Houri, S.; Hofmann, S.; van der Zant, H. S. J. Mechanical Characterization and Cleaning of CVD Single-Layer h-BN Resonators. npj 2D Mater. Appl. 2017, 1, 16.

(70) Piquemal-Banci, M.; Galceran, R.; Godel, F.; Caneva, S.; Martin, M.-B.; Weatherup, R. S.; Kidambi, P. R.; Bouzehouane, K.; Xavier, S.; Anane, A. Insulator-to-Metallic SpinFiltering in 2D-Magnetic Tunnel Junctions Based on Hexagonal Boron Nitride. $A C S$ Nano 2018.

(71) Lee, J. S.; Choi, S. H.; Yun, S. J.; Kim, Y. I.; Boandoh, S.; Park, J.-H.; Shin, B. G.; Ko, H.; Lee, S. H.; Kim, Y.-M. Wafer-Scale Single-Crystal Hexagonal Boron Nitride Film via Self-Collimated Grain Formation. Science 2018, 362, 817-821.

(72) Deemyad, S.; Silvera, I. F. Temperature Dependence of the Emissivity of Platinum in the IR. Rev. Sci. Instrum. 2008, 79, 10-12.

(73) Bluhm, H.; Havecker, M.; Knop-Gericke, A.; Kiskinova, M.; Schlogl, R.; Salmeron, M.; Hävecker, M.; Knop-Gericke, A.; Kiskinova, M.; Schlögl, R.; Salmeron, M. In Situ XRay Photoelectron Spectroscopy Studies of Gas-Solid Interfaces at near-Ambient Conditions. Mrs Bull. 2007, 32, 1022-1030.

(74) Zwick, A.; Carles, R. Multiple-Order Raman Scattering in Crystalline and Amorphous Silicon. Phys. Rev. B 1993, 48, 6024-6032. 


\section{Acknowledgements}

We acknowledge funding from the ERC (InsituNANO, grant 279342) and EPSRC (EP/K016636/1). R.W. acknowledges EPSRC Doctoral Training Award (EP/M506485/1). F.M. acknowledges funding from EPSRC Grant No. EP/P00945X/1. R.S.W. acknowledges funding from the European Union's Horizon 2020 research and innovation programme through an EU Marie Skłodowska-Curie Individual Fellowship (Global) under grant ARTIST (no. 656870). We wish to thank M.A.L. for proofreading.

\section{Additional Information}

\section{Supporting Information}

Further detailed information about the experimental procedure, the parameter space for growth and characterization is provided in the supplementary information. This material is available free of charge via the Internet at http://pubs.acs.org.

\section{Competing interests}

The authors declare no competing financial interests. 


\section{Figures}

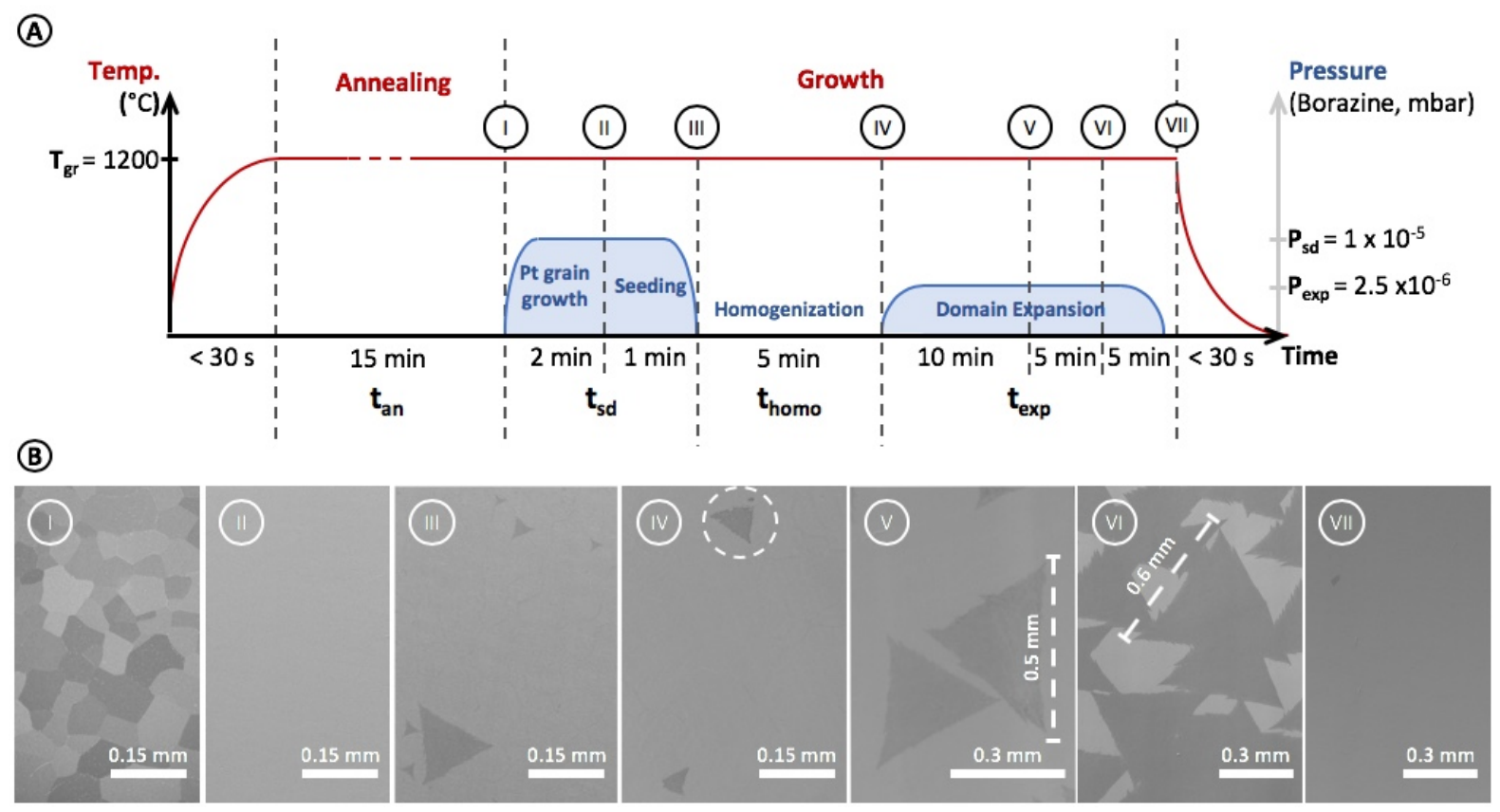

Figure 1 (a) Process flow diagram of SSG. The growth temperature is $\mathrm{T}_{\mathrm{gr}}=1200^{\circ} \mathrm{C}$. Precursor pressure during seeding is $\mathrm{P}_{\mathrm{sd}}=1 \times 10^{-5}$ mbar and $\mathrm{P}_{\mathrm{exp}}=2.5 \times 10^{-6}$ mbar during domain expansion (b) SEM images of $\mathrm{h}-\mathrm{BN}$ on Pt at different stages of growth. Growth was stopped at the respective stages, by removing the precursor and turning off the laser heating. In image IV, after annealing of nuclei, damage to existing domains is clearly visible (dotted outline). 

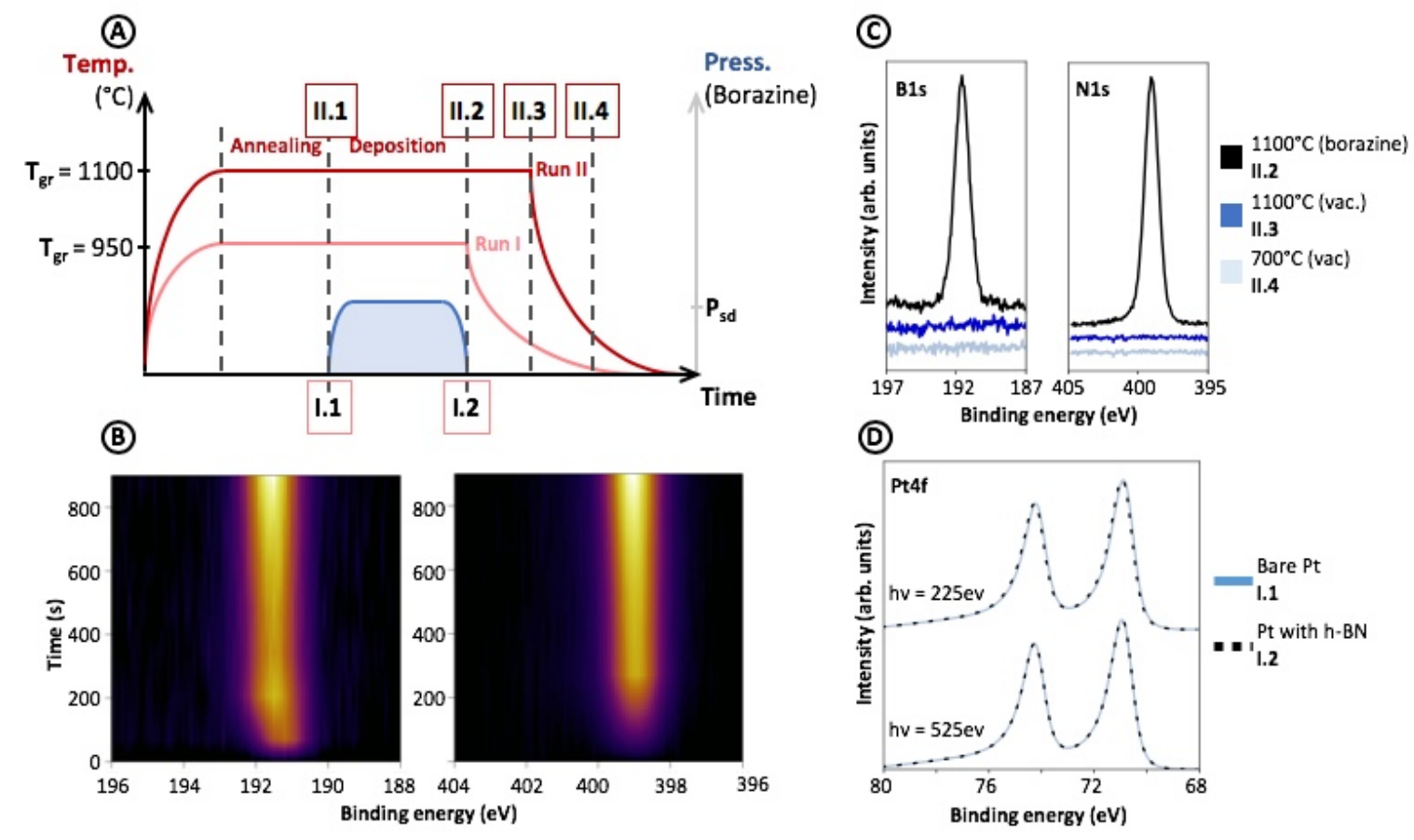

(2)

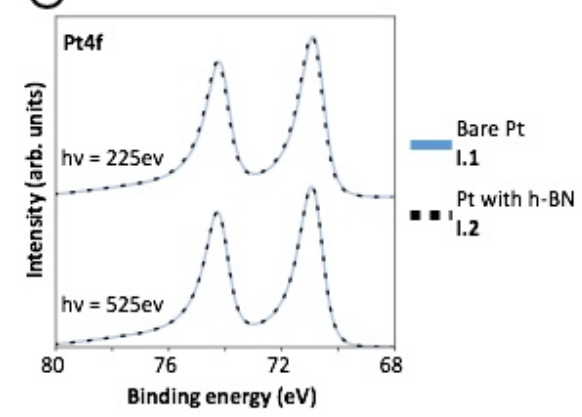

Figure 2 In situ XPS measurements during h-BN growth on Pt foil. Details on the conditions are given in the experimental section. (a) Schematic process flow diagram highlighting at which point of the process the spectra shown in (c), (d), and (e) were taken. (b) Evolution of the $\mathrm{B} 1 \mathrm{~s}$ and $\mathrm{N}$ 1s XP core level with borazine $\left(3 \times 10^{-4} \mathrm{mbar}\right)$ exposure time (spectra taken between II.1-II.2) for a Pt foil at $1100{ }^{\circ} \mathrm{C}$ (c) B 1s and N 1s XP spectra taken at an excitation energy of $h v=620 \mathrm{eV}$ for $\mathrm{T}_{\mathrm{gr}}=1100{ }^{\circ} \mathrm{C}$ with precursor present, $\mathrm{T}_{\mathrm{gr}}=1100^{\circ} \mathrm{C}$ in vacuum and RT in vacuum. The peak positions of B1s/N1s are $191.6 \mathrm{eV} / 399.0 \mathrm{eV}$. Shortly after removing borazine, the B/N peaks disappear and do not reappear during cooling. (d) Depth resolved Pt 4f XP spectra taken for Pt covered with h-BN and bare Pt at $\mathrm{T}_{\mathrm{gr}}=950^{\circ} \mathrm{C}$. No difference in peak positions and/or additional peaks are visible, confirming the absence of potential $\mathrm{Pt}$ compounds. 

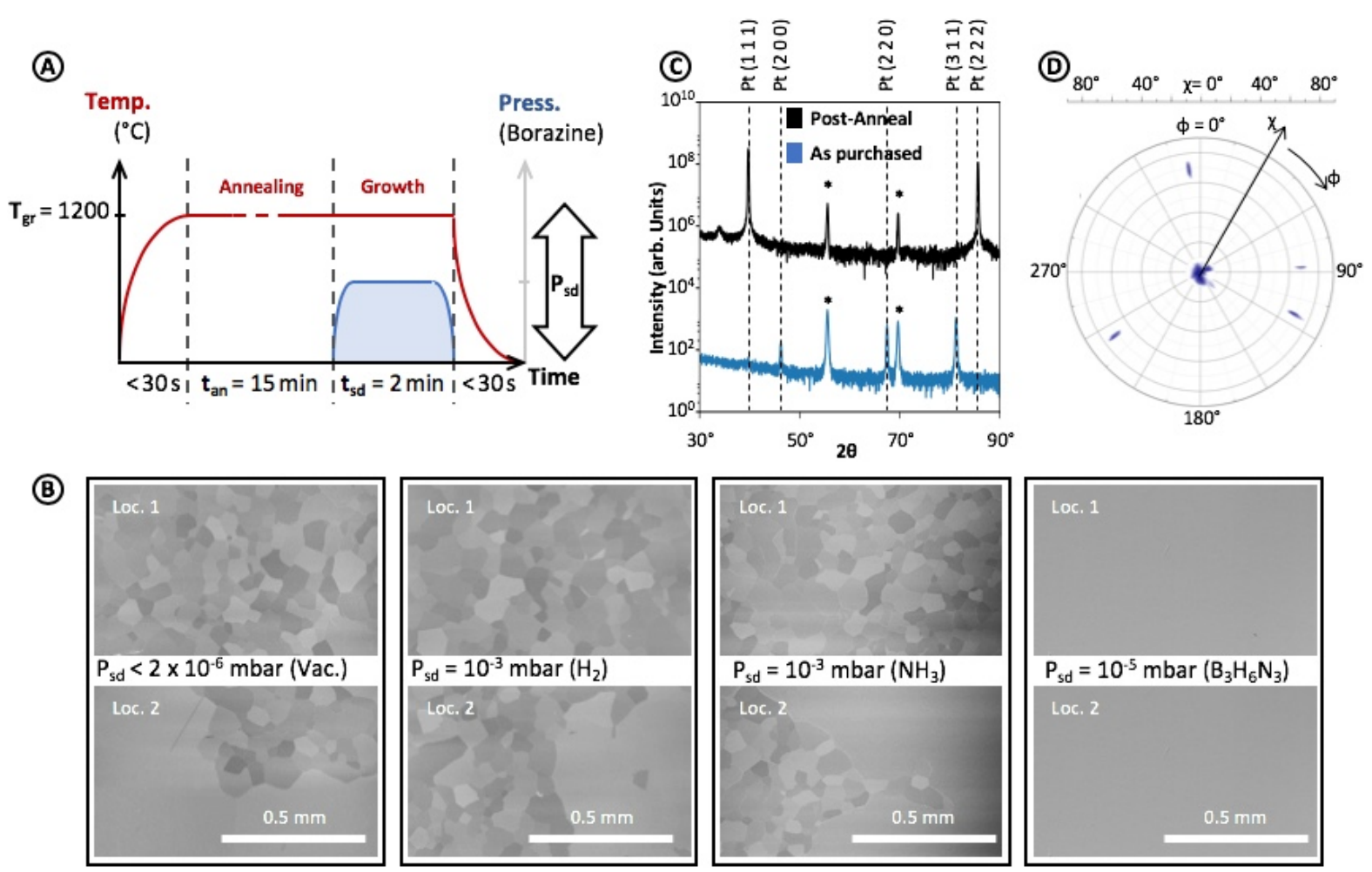

Figure 3 One-step process at identical $\mathrm{T}_{\mathrm{gr}}=1200^{\circ} \mathrm{C}$ for $2 \mathrm{~min}$, but with different gas environments. (a) Process flow diagram for recrystallization baseline experiment. All parameters were held constant, only type of gas and the pressure was varied. (b) SEM images of Pt foils after annealing. For each experimental condition, SEM images of two locations on the same substrate were provided to highlight the differences in texture. When annealing in vacuum, $\mathrm{H}_{2}$, or $\mathrm{NH}_{3}$, although certain polycrystalline regions remain (top images), it is apparent that the growth of large single crystal regions has occurred (bright region in bottom images). In contrast, the sample treated with borazine shows no polycrystalline regions. (c) XRD measurement of $\mathrm{Pt}$ foils as purchased and after recrystallization in borazine $\left(\mathrm{P}_{\mathrm{sc}}=10^{-5}\right.$ mbar, $\mathrm{T}_{\mathrm{gr}}=1200{ }^{\circ} \mathrm{C}, \mathrm{t}_{\mathrm{an}}=15 \mathrm{~min}$ and. $\mathrm{t}_{\mathrm{sd}}=2 \mathrm{~min}$ ). The spectra have been offset for better visibility (Post-Anneal spectrum was multiplied with a factor of $10^{4}$ ). All Pt related peaks are marked. The peaks marked with * originate from the Ta susceptor on which the Pt foil is mounted. While there are multiple orientations for the pristine foil, the dominating orientation 
is $\left(\begin{array}{lll}1 & 1 & 1\end{array}\right)$ after annealing. (d) Texture map of the $\mathrm{Pt}\left(\begin{array}{lll}1 & 1 & 1\end{array}\right)$ reflection at $2 \theta=39.73^{\circ}$. The Pt foil behaves like a single crystal, with one pole in the symmetric position $\left(\chi \sim 0^{\circ}\right)$ and 3 poles at $\chi$ $\sim 70^{\circ}$ and $\varphi=120^{\circ}$ apart from each other. A minor pole is visible at $\chi \sim 70^{\circ}$ and $\phi=90^{\circ}$, which indicates a minority of differently oriented grains. 


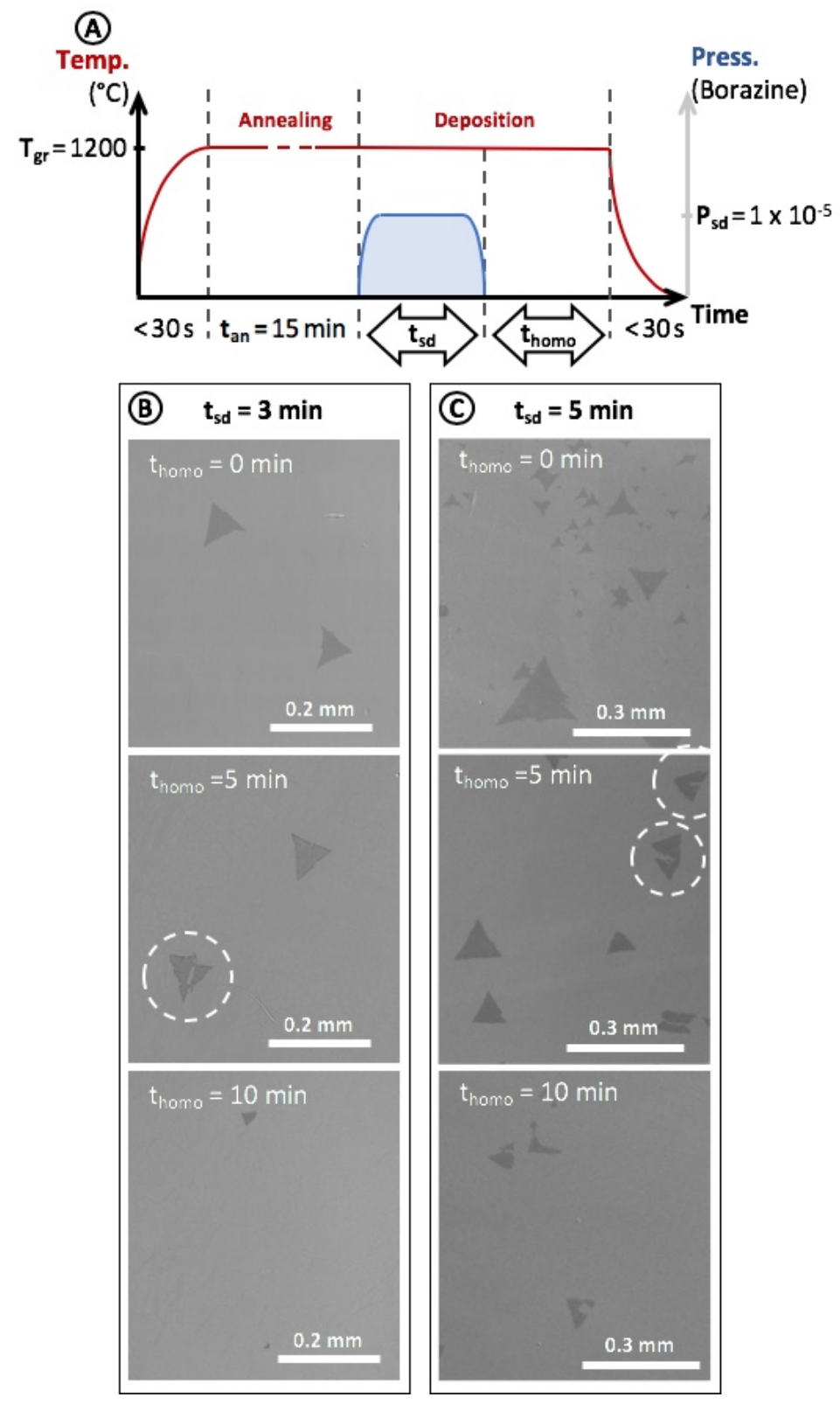

Figure 4 (a) Schematic process flow diagram for SG experiment to highlight the effect of seeding and homogenization. (b)-(c) SEM images of growth result. Growth was stopped at the respective stages, by removing the precursor and turning off the laser heating All parameters were kept constant, except of $t_{\text {sd }}$, which is varied between the series [3min in (e) and in $5 \mathrm{~min}(\mathrm{f})]$ and thomo, which is varied within each of the series. 

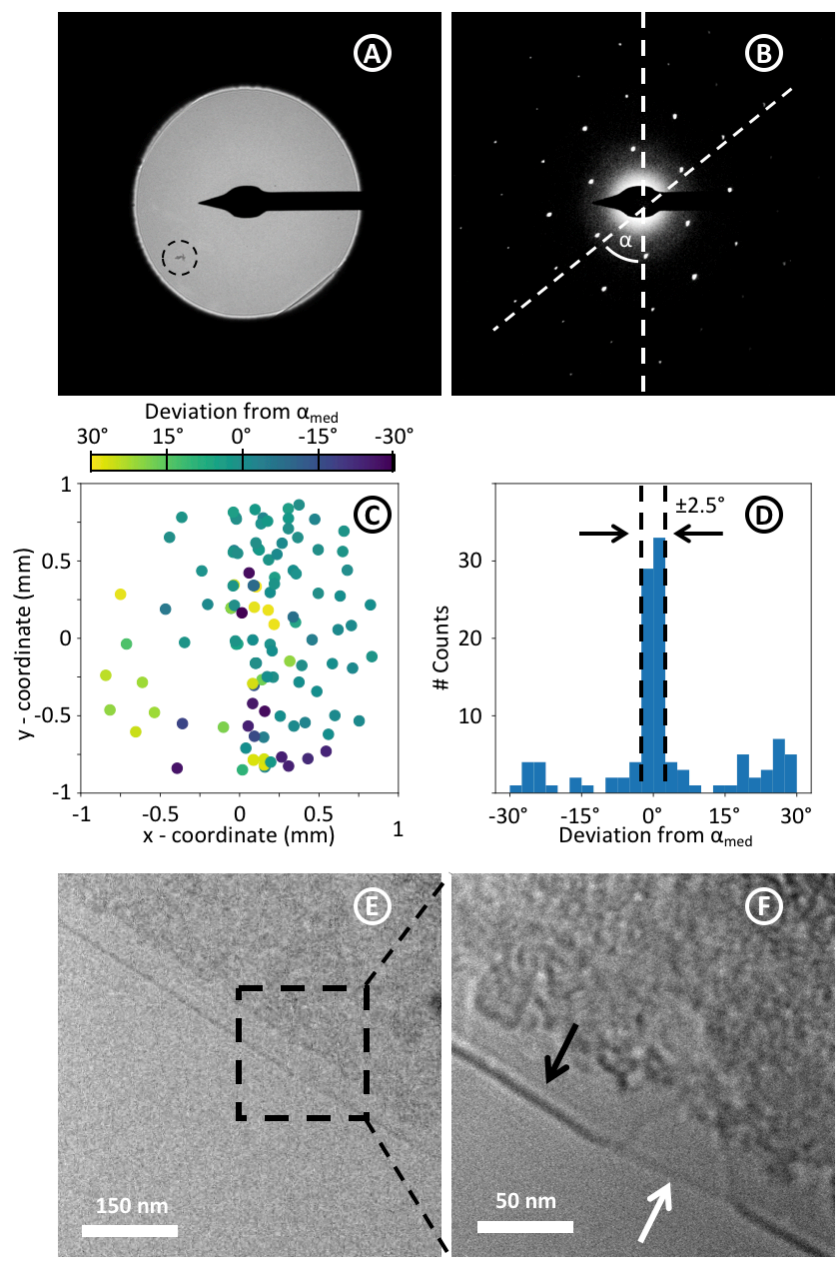

Figure 5 (a) BF-TEM images of h-BN. The dotted circle marks a particle on the suspended h$\mathrm{BN}$, which is otherwise indiscernable. (b) DF-TEM image corresponding to (a). $\alpha$ is defined as the angle between the vertical and the closest first order diffraction spot in clockwise direction. (c) Scatter map of the rotational deviation from $\alpha_{\text {med }}$ (median value of $\alpha$ ). (d) Distribution of orientation as deviation from $\alpha_{\text {med }}$ (e) \& (f) High magnification BF-TEM image of the edge of the h-BN film. Only one fringe is found (black arrow), which confirms the monolayer nature of BN. A small dent can be seen (white arrow) caused by folding of the layer. The contrast between single layer edge and folded edge is clearly visible. 
(A)

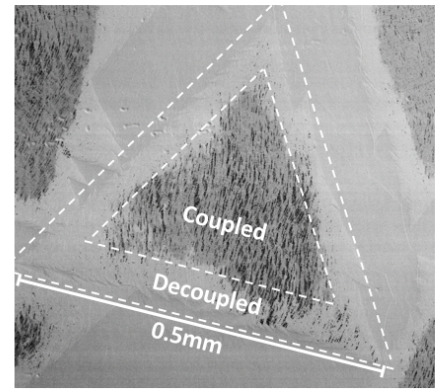

(B)
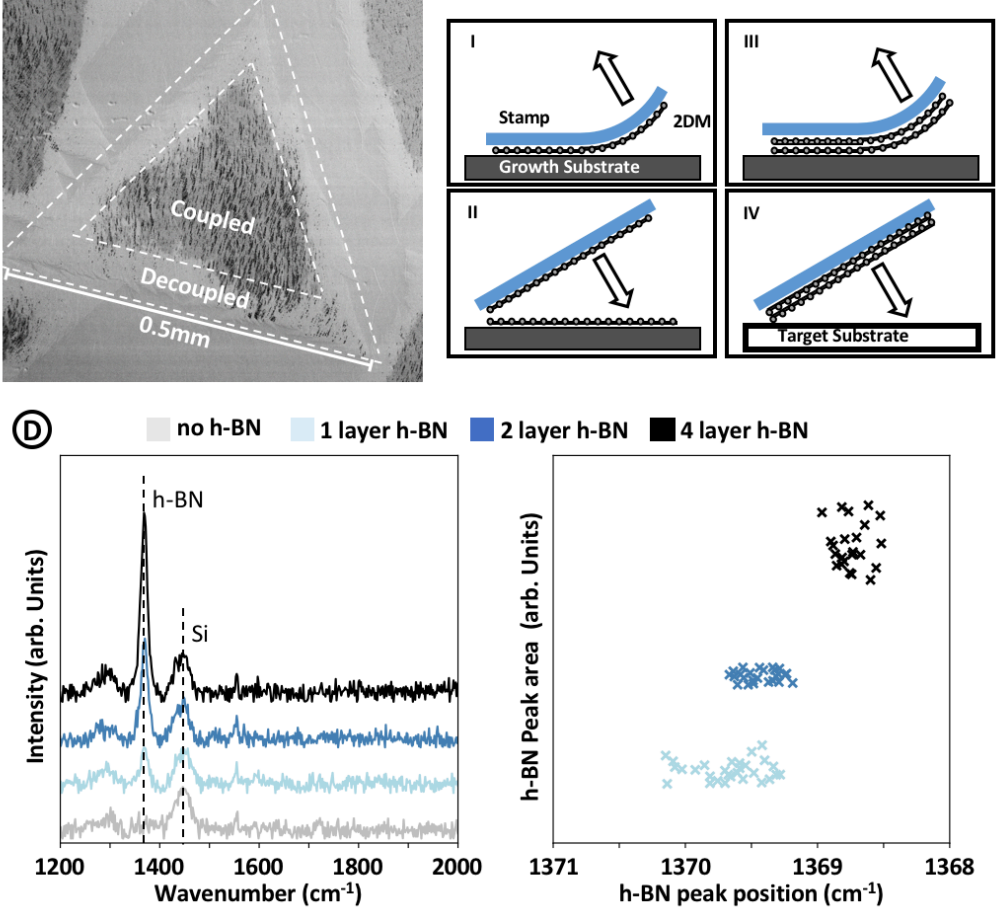

(C)
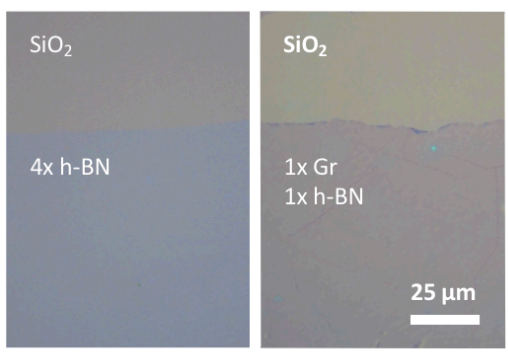

(ㄷ)

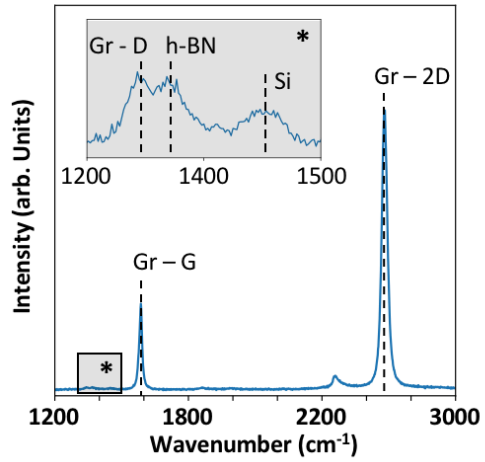

Figure 6 (a) SEM image of h-BN on Pt. The image is taken 5 hours after removing the sample from the reactor. The dotted lines mark the outer edge of the domains and the limit between the coupled (black) and decoupled (white) regions. (b) Process flow diagram of exfoliation based transfer. The PVA stamp is drop-cast onto the as-grown h-BN, which can then be peeled off and used for sequential exfoliation. After transfer onto the target substrate the stamp is dissolved in water (c) Optical images after transfer onto $\mathrm{SiO}_{2}$ of 4-layer h-BN and h-BN / Gr ( Gr in contact with $\left.\mathrm{SiO}_{2}\right)$ (d) The left graph shows the Raman spectrum of h$\mathrm{BN}$ after transfer onto $\mathrm{SiO}_{2}$ depending on the layer number. The spectra have been offset for better visibility. Si marks the $3^{\text {rd }}$ order silicon peak at $\sim 1450 \mathrm{~cm}^{-1} 74$. The peak at $\sim 1370 \mathrm{~cm}^{-1}$ corresponds to h-BN ${ }^{62}$. The right-hand plot presents the peak area after fitting with a Lorentzian curve, against the peak position for multiple measurements of different numbers of h-BN layers. For better visibility, only points between the $1^{\text {st }}$ and $3^{\text {rd }}$ quartile are shown for each sample. The median of the peak position is $1369.7 \mathrm{~cm}^{-1}, 1369.4 \mathrm{~cm}^{-1}, 1368.7 \mathrm{~cm}^{-1}$ and 
the median normalized peak area is 1, 2.24 and 4.03 for monolayer, bilayer and 4-layer h-BN.

(e) Raman spectrum of h-BN/Gr stack after transfer ( $\mathrm{Gr}$ in contact with $\left.\mathrm{SiO}_{2}\right)$. Inset shows magnified region to highlight the h-BN peak 
(A)
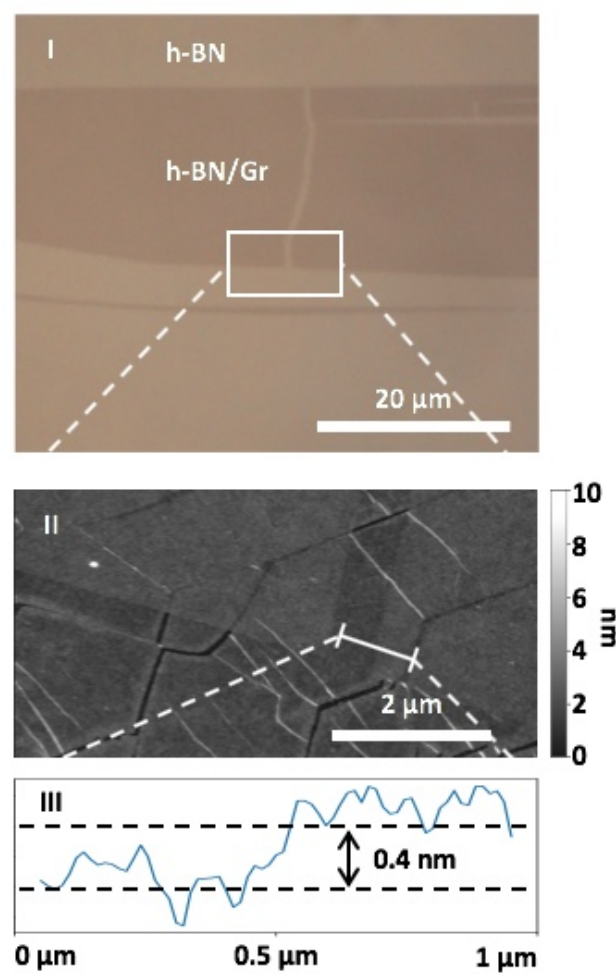

(B)
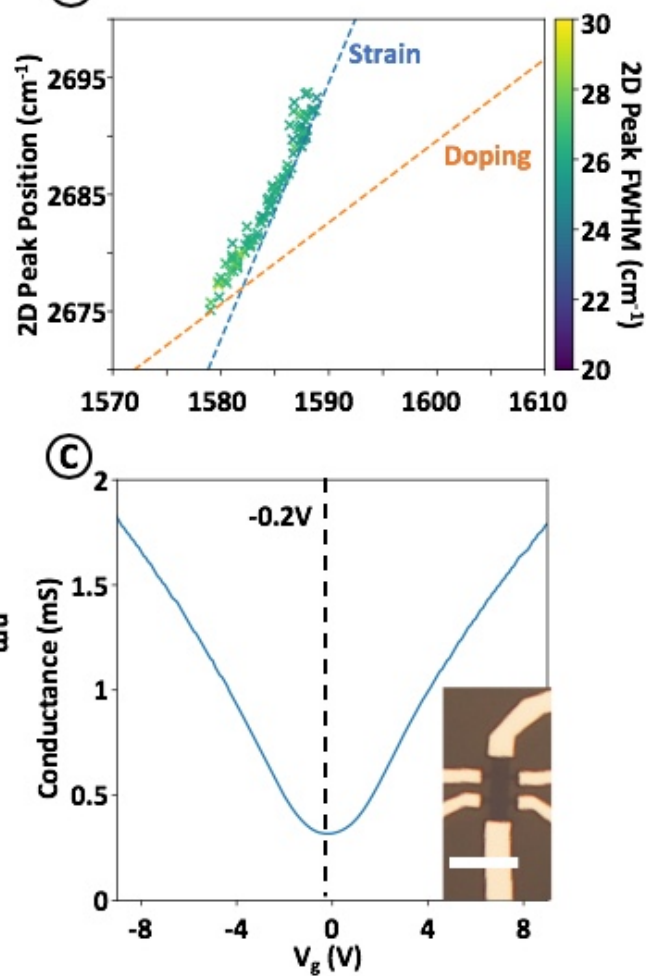

Figure 7 (a.I) Optical image of monolayer CVD h-BN on exfoliated Gr transferred onto a 90 $\mathrm{nm} \mathrm{SiO} 2 / \mathrm{Si}$ wafer ( $\mathrm{Gr}$ between $\mathrm{h}-\mathrm{BN}$ and $\mathrm{SiO}_{2}$ ). $\mathrm{h}$ - $\mathrm{BN}$ is not discernible as it uniformly covers the sample. (a.II) Peak force atomic force microscope (PF-AFM, details in experimental section) of area marked in (a.I). (a.III) Profile of line marked in (a.II). Step height of transition from h-BN only to h-BN/Gr region is about $\sim 0.4 \mathrm{~nm}$, as expected for single layer graphene, indicating a clean interface. (b) Peak position of the G- and 2D-peak of Gr measured by Raman spectroscopy. Colour of cross relates to FWHM of the 2D peak (See associated colour bar). The dotted blue line is the strain axis (slope 2.2), dotted orange line the doping axis (slope 0.7 ) and the charge neutrality point is $\left(1582 \mathrm{~cm}^{-1}, 2677 \mathrm{~cm}^{-1}\right) \cdot{ }^{66}$ (c) Transfer curve obtained via 4terminal measurement, details given in experimental methods section. The position of the Dirac point is marked. An optical image of the Hall bar measured is shown in the inset. The scale bar indicates $10 \mu \mathrm{m}$. 
TOC Image

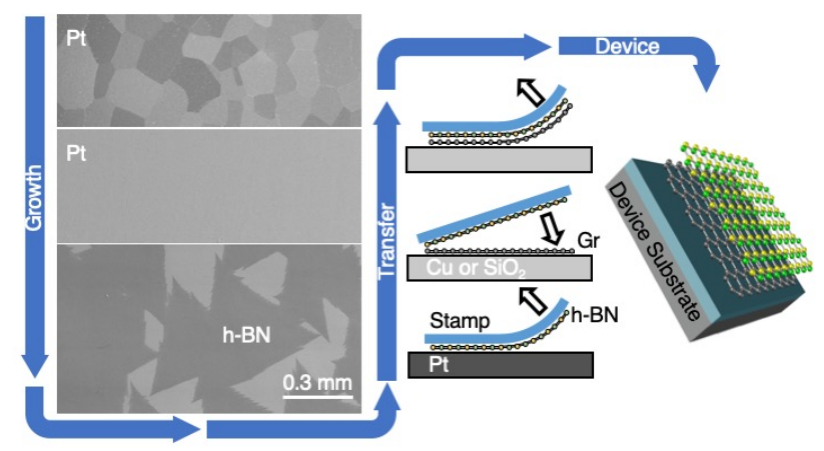

